\title{
LSP sneutrino novel decays
}

\author{
D. Aristizabal Sierra ${ }^{a, 1}$, D. Restrepo ${ }^{b, 2}$, S. Spinner ${ }^{c, 3}$ \\ ${ }^{a}$ IFPA, Dep. AGO, Universite de Liege, Bat B5, \\ Sart Tilman B-4000 Liege 1, Belgium. \\ ${ }^{b}$ Instituto de Fisica, Universidad de Antioquia, \\ A.A. 1226, Medellin, Colombia. \\ ${ }^{c}$ SISSA and INFN, Sezione di Trieste, \\ Via Bonomea 265, 34136 Trieste, Italy.
}

\begin{abstract}
In bilinear R-parity violation $(\mathrm{BRpV})$, in which the superpotential includes a bilinear term between the lepton doublet and the up-type Higgs superfields, a sneutrino LSP can decay into pairs of heavy standard model states $(W \mathrm{~s}, Z \mathrm{~s}$, tops or Higgs bosons), neutrinos or different-flavor charged lepton modes $\left(l_{i} l_{j}\right)$. These finals states can dominate over the traditionally considered bottom pair final state and would lead to unique and novel supersymmetric signals: multileptons events or pairs of heavy standard model fields. We investigate this possibility and find that the branching ratio into these states dominates when the bilinear term is much smaller than the sneutrino vacuum expectation value for a given sneutrino flavor. When $\mathrm{BRpV}$ is the only source of neutrino masses these decays can only dominate for one of the sneutrino generations. Relaxing this constraint opens these channels for all three generations.
\end{abstract}

\footnotetext{
${ }^{1}$ e-mail address: daristizabal@ulg.ac.be

${ }^{2}$ e-mail address: restrepo@udea.edu.co

${ }^{3}$ e-mail address: sspinner@sissa.it
} 


\section{Introduction}

As the large hadron collider (LHC) continues to successfully probe the nature of electroweak symmetry breaking (a recent breakthrough being the discovery of a Higgs candidate $[1,2]$ ), a solution to the gauge hierarchy problem, if it exists, has evaded our efforts so far. An elegant candidate for such a solution and one which addresses several other open issues as well, e.g. dark matter and gauge coupling unification, is supersymmetry (SUSY). Because of its theoretical appeal, it is important to understand all the guises that SUSY may adopt in order to recognize it if it is produced at the LHC.

An important open issue in SUSY, which has strong ramifications for its LHC phenomenology, as well as its cosmology, is the gauge invariance of lepton and baryon number violating interactions. Aside from introducing many new unknown parameters, these interactions also lead to rapid proton decay. The most common solution to address this issue is the imposing of a discrete symmetry, R-parity, defined as $R_{p} \equiv(-1)^{3(B-L)+2 S}$ (see [3] for a review). This forbids all tree-level lepton and baryon number violating terms and also causes the lightest supersymmetric particle (LSP) to be stable and therefore neutral. This LSP can then play the role of dark matter and would manifest itself at colliders as missing energy. However, proton decay requires the elimination of only the lepton number or baryon number violating terms and from a theoretical perspective, R-parity can be ad hoc. Furthermore, an open-mindedness to possible signals at the LHC should push us to consider alternatives. Finally, when R-parity is violated the stringent constraints on superpartner masses, derived from negative collider searches for missing energy events at the Tevatron and the $\mathrm{LHC}[4,5]$, can be relaxed ${ }^{1}$.

A systematic study of all possible R-parity violating terms and their effects on phenomenology is an arduous task and furthermore, one would like a mechanism for understanding why proton decay is significantly suppressed. A well-motivated solution to both of these issues are models which can predict the fate of R-parity. A natural framework for this endeavor is in the context of $U(1)_{B-L}$ symmetries, see $[9,10,11]$ for early examples ${ }^{2}$. While some $B-L$ models predict R-parity conservation [14, 15], the most minimal ones (in terms of particle content) require $\mathrm{R}$-parity violation $(\mathrm{RpV})[9,11,16,17,18]$. Even some non-minimal models prefer $\mathrm{RpV}$ from considerations of the renormalization group evolution of the soft masses [10, 19]. These and other models of spontaneous R-parity violation, such as [20,21], have the common feature that they can be described, in an effective field theory way, by bilinear R-parity violation $(\mathrm{BRpV})$ : the only R-parity violating terms are the mixings between the lepton doublets and up-type Higgs doublet in the superpotential. This makes BRpV a powerful tool for studying possible signatures of spontaneous RpV. Furthermore, proton decay is highly suppressed and it is important to mention that a gravitino LSP can be a dark matter candidate in such models [21, 22].

Once R-parity is broken the LSP is no longer stable and therefore astrophysical constraints on its nature do not apply [23]. Accordingly, from a purely phenomenological

\footnotetext{
${ }^{1}$ See ref. $[6,7,8]$ for details.

${ }^{2}$ Horizontal symmetries $U(1)_{X}$ can be also used to construct models where the $\mathrm{RpV}$ couplings, arising from effective operators, are intrinsically small $[12,13]$.
} 
point of view any superpartner can be the LSP, and studies of the different possibilities in BRpV models (and of sleptons and sneutrinos in general models [24, 25]) and their relation to neutrino masses and mixings have been carried out in e.g. [26, 27, 28, 29].

In this paper we extend upon previous results by considering sneutrino $\mathrm{LSP}^{3}$ decays in BRpV into "non-conventional" modes: pairs of heavy standard model (SM) states $\left(W^{+} W^{-}, Z^{0} Z^{0}, h^{0} h^{0}, t \bar{t}\right)$, invisible modes $(\nu \nu)$ and different-flavor charged lepton final states $\left(l_{i}^{+} l_{j}^{-}, i \neq j\right)$. Such states, to our knowledge, had not received much attention before despite the fact that they can dominate sneutrino decays and can yield unique and unanticipated SUSY signals ${ }^{4}$. Specifically, neutrino masses force RpV to be small so its only effect is on the decay of the LSP. Therefore, for a sneutrino LSP, every SUSY event will eventually decay into two sneutrinos which could then decay into one of the aforementioned states. As we will show, this typically translates into into different-flavor charged leptons or heavy SM modes, depending on the sneutrino generation.

The main goal of this paper is to study the sneutrino decays into these "non-conventional" modes and to show that they can dominate over the traditionally considered $b \bar{b}$ final state. We find that the latter dominate roughly when the BRpV term, $\epsilon_{i}$ is smaller than the vacuum expectation value (vev) of the sneutrino, $v_{i}$, for a given flavor of sneutrino, $i$. In the case when BRpV is the only source of neutrino masses, this possibility can only hold for one sneutrino flavor, however, when this assumption is relaxed, it can hold true for all three generations. Therefore, if two or more generations of sneutrinos decay via $\mathrm{RpV}$, it might be possible to rule out $\mathrm{BRpV}$ as the sole generator of neutrino masses.

The rest of the paper is organized as follows. In section 2 we discuss the generalities of the BRpV model, in particular those related with the neutral scalar sector. In section 3 we derive formulas for $\mathrm{BRpV}$ induced mixings. In section 4 we write the relevant couplings for BRpV sneutrino decays, give analytical formulas for the different partial decay widths, analyze the constraints on parameter space enforced by neutrino data and present our results. In section 5 we summarize and present our conclusions.

\section{Bilinear R-parity violation}

In what follows we will briefly describe the main features of the bilinear R-parity breaking model, in particular those related with the neutral scalar sector. We shall closely follow the notation used in [32] and assume that all parameters are real ${ }^{5}$. Throughout the text matrices will be denoted in bold-face.

In addition to the MSSM R-parity conserving superpotential (where we have suppressed relevant indices):

$$
W_{\mathrm{MSSM}}=\boldsymbol{h}^{\boldsymbol{U}} \hat{Q} \hat{H}_{u} \hat{u}^{c}+\boldsymbol{h}^{\boldsymbol{D}} \hat{Q} \hat{H}_{d} \hat{d}^{c}+\boldsymbol{h}^{\boldsymbol{E}} \hat{L} \hat{H}_{d} \hat{e}^{c}+\mu \hat{H}_{u} \hat{H}_{d}
$$

\footnotetext{
${ }^{3}$ A sneutrino NLSP with a gravitino LSP would not change our phenomenological results.

${ }^{4}$ Gauge boson and top quark pair production through a sneutrino resonance in trilinear $\mathrm{RpV}$ was studied in [30] while the full set of heavy SM modes have been mentioned in BRpV models in [31]

${ }^{5}$ This simplification does not affect our main conclusions.
} 
the bilinear R-parity breaking model also contains the following terms:

$$
W_{\mathrm{BRpV}}=\epsilon_{\alpha \beta} \epsilon_{i} \hat{L}_{i}^{\alpha} \hat{H}_{u}^{\beta}
$$

where $\epsilon_{\alpha \beta}$ is the $S U(2)$ completely antisymmetric tensor, $i=1,2,3$ runs over the SM fermion generations and $\epsilon_{i}$ is the R-parity and lepton number breaking bilinear parameter with units of mass. Consistency then requires a new set of soft SUSY breaking terms in the scalar potential, namely

$$
V_{\mathrm{BRpV}}=B_{i} \epsilon_{i} \epsilon_{\alpha \beta} \tilde{L}_{i}^{\alpha} H_{u}^{\beta}
$$

Neglecting soft flavor mixing, the scalar potential relevant for neutral scalars is

$$
\begin{aligned}
V & \supset\left(m_{H_{d}}^{2}+\mu^{2}\right) H_{d}^{\dagger} H_{d}+\left(m_{H_{u}}^{2}+\mu^{2}\right) H_{u}^{\dagger} H_{u}+m_{L_{i}}^{2} \tilde{L}_{i}^{\dagger} \tilde{L}_{i} \\
& +\frac{1}{8} g_{Z}^{2}\left(H_{u}^{\dagger} H_{u}-H_{d}^{\dagger} H_{d}-\tilde{L}_{i}^{\dagger} \tilde{L}_{i}\right)^{2}+|\boldsymbol{\epsilon}|^{2} H_{u}^{\dagger} H_{u}+\epsilon_{i} \epsilon_{j} \tilde{L}_{i}^{\dagger} \tilde{L}_{j} \\
& +\left(-\mu \epsilon_{i} \tilde{L}_{i}^{\dagger} H_{d}-B \mu \epsilon_{\alpha \beta} H_{d}^{\alpha} H_{u}^{\beta}+B_{i} \epsilon_{i} \epsilon_{\alpha \beta} \tilde{L}_{i}^{\alpha} H_{u}^{\beta}+\text { H.c. }\right),
\end{aligned}
$$

with $g_{Z}^{2}=g^{2}+g^{\prime 2}$ and $\boldsymbol{\epsilon}^{T}=\left(\epsilon_{1}, \epsilon_{2}, \epsilon_{3}\right)$. Electroweak symmetry is broken once the Higgs and slepton acquire a vev, $\left\langle H_{d, u}\right\rangle=v_{d, u} / \sqrt{2}$ and $\left\langle\tilde{L}_{i}\right\rangle=v_{i} / \sqrt{2}$, with $v=\left(v_{u}^{2}+v_{d}^{2}+\right.$ $\left.\sum_{i=1,2,3} v_{i}^{2}\right)^{1 / 2} \simeq 246 \mathrm{GeV}$ and $M_{Z}^{2}=g_{Z}^{2} v^{2} / 4$. The doublets are parameterized as

$$
H_{d}=\left(\begin{array}{c}
H_{d}^{0} \\
H_{d}^{-}
\end{array}\right), \quad H_{u}=\left(\begin{array}{c}
H_{u}^{+} \\
H_{u}^{0}
\end{array}\right), \quad \tilde{L}_{i}=\left(\begin{array}{c}
\tilde{L}_{i} \\
l_{i}^{-}
\end{array}\right)
$$

with the neutral components given by

$$
H_{d}^{0}=\frac{1}{\sqrt{2}}\left(\sigma_{d}^{0}+i \varphi_{d}^{0}+v_{d}\right), \quad H_{u}^{0}=\frac{1}{\sqrt{2}}\left(\sigma_{u}^{0}+i \varphi_{u}^{0}+v_{u}\right), \quad \tilde{L}_{i}=\frac{1}{\sqrt{2}}\left(\tilde{\nu}_{i}^{R}+i \tilde{\nu}_{i}^{I}+v_{i}\right)
$$

Here we introduce the notation $\tilde{\nu}^{R, I}$ to differentiate the CP-even sneutrinos from the CP-odd.

In the basis $\left(S^{0}\right)^{T}=\left(\sigma_{d}^{0}, \sigma_{u}^{0}, \tilde{\nu}_{i}^{R}\right)^{T}$ the linear part of the neutral scalar potential can be written as

$$
V_{\text {linear }}\left(S_{i}^{0}\right)=\sum_{a=1, \cdots, 5} t_{a} S_{a}^{0}
$$

where the $t_{a}$ 's are the so-called tadpoles. At tree-level these are (see eqs. (4) and (7))

$$
\begin{aligned}
& t_{d}^{(0)}=\left(m_{H_{d}}^{2}+\mu^{2}\right) v_{d}+D v_{d}-\mu\left(B v_{u}+\boldsymbol{\epsilon} \cdot \boldsymbol{v}\right) \\
& t_{u}^{(0)}=-B \mu v_{d}+\left(m_{H_{u}}^{2}+\mu^{2}\right) v_{u}-D v_{u}+\sum_{i=1,2,3} B_{i} \epsilon_{i} v_{i}+|\boldsymbol{\epsilon}|^{2} v_{u}, \\
& t_{i}^{(0)}=D v_{i}+\epsilon_{i}\left(-\mu v_{d}+B_{i} v_{u}+\boldsymbol{\epsilon} \cdot \boldsymbol{v}\right)+m_{L_{i}}^{2} v_{i}
\end{aligned}
$$

where $\boldsymbol{v}^{T}=\left(v_{1}, v_{2}, v_{3}\right)$ and $D=g_{Z}^{2}\left(v_{d}^{2}-v_{u}^{2}+\sum_{i} v_{i}^{2}\right) / 8$. The minimization of the potential, $V_{\text {linear }}=0$, requires the tadpoles to vanish. Thus, the vevs can be determined from the 
system of equations in (8) by imposing $t_{a}=0(a=1, \ldots, 5)$. In particular, considering only leading order BRpV terms, the sneutrino vevs can be written as

$$
v_{i} \simeq \frac{\epsilon_{i} v}{m_{\tilde{\nu}_{i}}^{2}}\left(\mu c_{\beta}-B_{i} s_{\beta}\right)
$$

where $m_{\tilde{\nu}_{i}}^{2}=m_{L_{i}}^{2}+M_{Z}^{2}\left(c_{\beta}^{2}-s_{\beta}^{2}\right) / 2$ (the tree level sneutrino mass) and $t_{\beta} \equiv \tan \beta=v_{u} / v_{d}$.

\section{$3 \quad$ R-parity violating mixings}

Without conserved R-parity, there are no quantum numbers to distinguish the leptons and sleptons from the gauginos and Higgsinos and Higgs bosons respectively. Therefore, in BRpV several mixings between supersymmetric and non supersymmetric particles exist: (i) neutralinos mix with neutrinos, (ii) charginos mix with charged leptons, (iii) Higgs bosons mix with the sneutrinos and $(i v)$ charged Higss bosons mix with charged sleptons. These mixings are important for calculating LSP decays, especially mixings of type $(i)$ as they allow to fix - via experimental neutrino data - the size of the BRpV parameters. Since mixings of type $(i v)$ are not of interest for sneutrino decays, in what follows we will only discuss analytical approximations for mixings of type $(i)-(i i i)$.

\subsection{Neutralino-neutrino mixings}

In the basis $\left(\psi^{0}\right)^{T}=\left(-i \lambda,-i \lambda_{3}, \tilde{H}_{d}^{0}, \tilde{H}_{u}^{0}, \nu_{i}\right)$ the neutral fermion mass matrix can be written as

$$
\mathcal{L}_{\psi^{0}}=-\frac{1}{2}\left(\psi^{0}\right)^{T} \boldsymbol{M}_{\boldsymbol{N}} \psi^{0}+\text { H.c. }
$$

with

$$
\boldsymbol{M}_{\boldsymbol{N}}=\left(\begin{array}{cc}
\left.\boldsymbol{M}_{\chi^{0}}\right|_{4 \times 4} & \left.\boldsymbol{M}_{\chi \nu}^{T}\right|_{4 \times 3} \\
\left.\boldsymbol{M}_{\boldsymbol{\chi}}\right|_{3 \times 4} & \left.\mathbf{0}\right|_{3 \times 3}
\end{array}\right),
$$

where $\boldsymbol{M}_{\boldsymbol{\chi}}$ is the neutralino mass matrix:

$$
\boldsymbol{M}_{\chi^{0}}=\left(\begin{array}{cccc}
M_{1} & 0 & -g^{\prime} v_{d} / 2 & g^{\prime} v_{u} / 2 \\
0 & M_{2} & g v_{d} / 2 & -g v_{u} / 2 \\
-g^{\prime} v_{d} / 2 & g v_{d} / 2 & 0 & -\mu \\
g^{\prime} v_{u} / 2 & -g v_{u} / 2 & -\mu & 0
\end{array}\right)
$$

and $M_{1}$ and $M_{2}$ are the soft masses for the bino and wino respectively. $\boldsymbol{M}_{\boldsymbol{\chi} \boldsymbol{\nu}}$ is the $4 \times 3$ neutralino-neutrino mixing matrix given by

$$
M_{\chi \nu}=\left(\begin{array}{llll}
-\frac{1}{2} g^{\prime} v_{i} & \frac{1}{2} g v_{i} & 0 & \epsilon_{i}
\end{array}\right) .
$$

In the Weyl mass eigenstate basis, defined as ${ }^{6}$

$$
F^{0}=\mathbf{N} \psi^{0},
$$

\footnotetext{
${ }^{6}$ The Majorana mass eigenstates are defined as $\overline{\chi^{0}}=\left(\overline{F_{i}^{0}} F_{i}^{0}\right)$.
} 
the mass matrix becomes

$$
\hat{M}_{N}=N^{*} M_{N} N^{\dagger}
$$

Due to the smallness of the BRpV parameters, at order $\epsilon_{i}, \boldsymbol{M}_{\boldsymbol{N}}$ can be block diagonalized by decomposing the diagonalizing matrix $\boldsymbol{N}$ as follows [32]:

$$
\boldsymbol{N}=\mathcal{N} \boldsymbol{\Xi} \simeq\left(\begin{array}{cc}
\boldsymbol{N}_{\boldsymbol{C}} & \mathbf{0} \\
\mathbf{0} & \boldsymbol{U}_{\boldsymbol{\ell}}^{\dagger}
\end{array}\right)\left(\begin{array}{cc}
\mathbb{I} & \boldsymbol{\xi}^{T} \\
-\boldsymbol{\xi}^{*} & \mathbb{I}
\end{array}\right)=\left(\begin{array}{cc}
\boldsymbol{N}_{\boldsymbol{C}} & \boldsymbol{N}_{\boldsymbol{C}} \boldsymbol{\xi}^{T} \\
-\boldsymbol{U}_{\boldsymbol{\ell}}^{T} \boldsymbol{\xi} & \boldsymbol{U}_{\boldsymbol{\ell}}^{T}
\end{array}\right)
$$

The matrix $\boldsymbol{\Xi}$ block diagonalizes $\boldsymbol{M}_{\boldsymbol{N}}$ to the form $\operatorname{diag}\left(\boldsymbol{M}_{\boldsymbol{\chi}^{\mathbf{0}}}, \boldsymbol{m}_{\boldsymbol{\nu}}^{\mathrm{eff}}\right)$, where $\boldsymbol{m}_{\boldsymbol{\nu}}^{\text {eff }}$ is the tree-level light neutrino mass matrix. The mixing parameters $\xi_{i j}$ can thus be determined to be

$$
\begin{array}{ll}
\xi_{i 1}=\frac{g^{\prime} M_{2} \mu}{2\left|\boldsymbol{M}_{\chi^{0}}\right|} \Lambda_{i}, & \xi_{i 2}=-\frac{g M_{1} \mu}{2\left|\boldsymbol{M}_{\chi^{0}}\right|} \Lambda_{i} \\
\xi_{i 3}=-\frac{\epsilon_{i}}{\mu}+\frac{\left(g^{2} M_{1}+g^{2} M_{2}\right) v_{u}}{4\left|\boldsymbol{M}_{\chi^{0}}\right|} \Lambda_{i}, & \xi_{i 4}=-\frac{\left(g^{2} M_{1}+g^{2} M_{2}\right) v_{u}}{4\left|\boldsymbol{M}_{\chi^{0}}\right|} \Lambda_{i}
\end{array}
$$

where $\left|\boldsymbol{M}_{\chi^{0}}\right|=-\left(M_{1} M_{2} \mu^{2}-2 M_{1} \mu M_{W}^{2} c_{\beta} s_{\beta}-2 M_{2} \mu M_{W}^{2} c_{\beta} s_{\beta} t_{\theta_{W}}\right)\left(\right.$ with $t_{W}=\tan \theta_{W}$, $\theta_{W}$ being the weak mixing angle) and

$$
\Lambda_{i}=\mu v_{i}+v_{d} \epsilon_{i}
$$

Finally the block diagonal mixing matrices $\boldsymbol{N}_{\boldsymbol{C}}$ and $\boldsymbol{U}_{\boldsymbol{\ell}}$ in (16) diagonlize the neutralino and neutrino effective mass matrix, which reads

$$
\left(\boldsymbol{m}_{\nu}^{\mathrm{eff}}\right)_{i j}=\frac{M_{1} g^{2}+M_{2} g^{2}}{4\left|\boldsymbol{M}_{\chi^{\mathbf{0}}}\right|} \Lambda_{i} \Lambda_{j}
$$

Since this matrix has two vanishing eigenvalues it can be diagonalized by only two rotation matrices, namely

$$
\boldsymbol{U}_{\ell}=\boldsymbol{U}_{\ell}\left(\theta_{23}^{\mathrm{BRpV}}\right) \boldsymbol{U}_{\ell}\left(\theta_{13}^{\mathrm{BRpV}}\right)
$$

with

$$
\tan ^{2} \theta_{23}^{\mathrm{BRpV}}=\frac{\Lambda_{2}^{2}}{\Lambda_{3}^{2}}, \quad \tan ^{2} \theta_{13}^{\mathrm{BRpV}}=\frac{\Lambda_{1}^{2}}{\Lambda_{2}^{2}+\Lambda_{3}^{2}} .
$$

For sneutrino decays the relevant part of the $\chi-\nu$ mixing turns out to be the $\boldsymbol{U}_{\ell}^{T} \boldsymbol{\xi}$ block, that from eqs. (17) and (20) can be written as [33]

$$
\boldsymbol{U}_{\ell}^{T} \boldsymbol{\xi}=\left(\begin{array}{cccc}
0 & 0 & -\bar{\epsilon}_{1} / \mu & 0 \\
0 & 0 & -\bar{\epsilon}_{2} / \mu & 0 \\
a_{1}|\boldsymbol{\Lambda}| & a_{2}|\boldsymbol{\Lambda}| & -\bar{\epsilon}_{3} / \mu & a_{4}|\boldsymbol{\Lambda}|
\end{array}\right)
$$

where $\bar{\epsilon}_{1,2}=\left(\boldsymbol{U}_{\ell}^{T}\right)_{(1,2) j} \epsilon_{j}$ and $\bar{\epsilon}_{3}=\tilde{\epsilon}_{3}-a_{3}|\boldsymbol{\Lambda}| \mu$ with $\tilde{\epsilon}_{3}=\left(\boldsymbol{U}_{\ell}^{T}\right)_{3 j} \epsilon_{j}$. The coefficients $a_{i}$ are given by

$$
a_{1}=\frac{g^{\prime} M_{2} \mu}{2\left|\boldsymbol{M}_{\chi^{0}}\right|}, a_{2}=-\frac{g M_{1} \mu}{2\left|\boldsymbol{M}_{\chi^{0}}\right|}, a_{3}=\frac{M_{\tilde{\gamma}} v s_{\beta}}{4\left|\boldsymbol{M}_{\chi^{0}}\right|}, a_{4}=-\frac{M_{\tilde{\gamma}} v c_{\beta}}{4\left|\boldsymbol{M}_{\boldsymbol{\chi}^{0}}\right|}
$$


with $M_{\tilde{\gamma}}=g^{2} M_{1}+g^{\prime 2} M_{2}$. Taking into account eqs. (20) and (21), explicitly $\bar{\epsilon}_{i}(i=1,2)$ and $\tilde{\epsilon}_{3}$ are given by

$$
\begin{aligned}
& \bar{\epsilon}_{1}=\frac{\epsilon_{1}\left(\Lambda_{2}^{2}+\Lambda_{3}^{2}\right)-\Lambda_{1}\left(\Lambda_{2} \epsilon_{2}+\Lambda_{3} \epsilon_{3}\right)}{|\boldsymbol{\Lambda}| \sqrt{\Lambda_{2}^{2}+\Lambda_{3}^{2}}}, \\
& \bar{\epsilon}_{2}=\frac{\Lambda_{3} \epsilon_{2}-\Lambda_{2} \epsilon_{3}}{\sqrt{\Lambda_{2}^{2}+\Lambda_{3}^{2}}} \\
& \tilde{\epsilon}_{3}=\frac{\boldsymbol{\Lambda} \cdot \boldsymbol{\epsilon}}{|\boldsymbol{\Lambda}|}
\end{aligned}
$$

\subsection{Chargino-charged lepton mass matrices and mixings}

In the bases $\left(\psi^{ \pm}\right)^{T}=\left(-i \lambda^{ \pm}, H_{u, d}^{ \pm}, e_{R, L}^{ \pm}\right)$the chargino charged lepton mass matrix is determined by the following Lagrangian

$$
-\mathcal{L}_{\psi^{ \pm}}=\frac{1}{2} \Psi^{T}\left(\begin{array}{cc}
\mathbf{0} & \boldsymbol{M}_{\boldsymbol{C}}^{T} \\
\boldsymbol{M}_{\boldsymbol{C}} & \mathbf{0}
\end{array}\right) \Psi+\text { H.c. },
$$

where $\Psi^{T}=\left(\psi^{+}, \psi^{-}\right)^{T}$ and in the basis in which the charged lepton mass matrix is diagonal $\boldsymbol{M}_{\boldsymbol{C}}$ can be written as

$$
M_{\boldsymbol{C}}=\left(\begin{array}{cc}
\left.\boldsymbol{M}_{\boldsymbol{\chi}}\right|_{2 \times 2} & \left.\boldsymbol{M}_{\boldsymbol{R} \chi}\right|_{2 \times 3} \\
\boldsymbol{M}_{\left.\boldsymbol{L} \chi\right|_{3 \times 2}} & \left.\hat{\boldsymbol{M}}_{\boldsymbol{\ell}}\right|_{3 \times 3}
\end{array}\right) .
$$

The block diagonal matrices correspond to the MSSM chargino and charged lepton mass matrices whereas the off-diagonal mass matrices read

$$
\boldsymbol{M}_{\boldsymbol{R \chi}}=\left(\begin{array}{c}
0 \\
-\frac{1}{\sqrt{2}} h_{i}^{E} v_{i}
\end{array}\right), \quad \boldsymbol{M}_{\boldsymbol{L \chi}}=\left(\begin{array}{ll}
\frac{1}{\sqrt{2}} g v_{i} & -\epsilon_{i}
\end{array}\right) .
$$

Defining the Weyl mass eigenstates as ${ }^{7}$

$$
F^{-}=\boldsymbol{U} \psi^{-} \quad \text { and } \quad F^{+}=\boldsymbol{V} \psi^{+},
$$

the diagonal mass matrix $\hat{M}_{C}$ is obtained through the biunitary transformation:

$$
\boldsymbol{U} \boldsymbol{M}_{\boldsymbol{C}} \boldsymbol{V}^{T}
$$

Approximate analytical expressions for the mixing matrices $\boldsymbol{U}, \boldsymbol{V}$ have been discussed in $[33,34,35]$. Here we describe the method for completeness.

The off-diagonal block matrix $\boldsymbol{M}_{\boldsymbol{R} \boldsymbol{\chi}}$, being proportional to the charged lepton Yukawa couplings, can be neglected, and due to the smallness of the BRpV parameters the mixing

\footnotetext{
${ }^{7}$ The corresponding Dirac eigenstates are defined as $\overline{\chi_{i}^{-}}=\left(\overline{F_{i}^{-}} F_{i}^{+}\right)$.
} 
matrices $\boldsymbol{U}, \boldsymbol{V}$ can be written according to

$$
\begin{gathered}
U=\mathcal{U} \Xi_{L} \simeq\left(\begin{array}{cc}
U_{L} & 0 \\
0 & \mathbb{I}
\end{array}\right)\left(\begin{array}{cc}
\mathbb{I} & \xi_{L}^{\dagger} \\
-\xi_{L} & \mathbb{I}
\end{array}\right), \\
V=\mathcal{V} \Xi_{R} \simeq\left(\begin{array}{cc}
V_{R} & 0 \\
0 & \mathbb{I}
\end{array}\right)\left(\begin{array}{cc}
\mathbb{I} & \xi_{R}^{T} \\
-\xi_{R}^{*} & \mathbb{I}
\end{array}\right),
\end{gathered}
$$

where, in first approximation in the BRpV parameters, the matrices $\boldsymbol{\Xi}_{\boldsymbol{L}, \boldsymbol{R}}$ block-diagonalize the mass matrix, namely

$$
\left(\begin{array}{cc}
\mathbb{I} & \boldsymbol{\xi}_{L}^{\dagger} \\
-\boldsymbol{\xi}_{L} & \mathbb{I}
\end{array}\right)\left(\begin{array}{cc}
M_{\chi} & 0 \\
M_{L \chi} & \hat{M}_{\ell}
\end{array}\right)\left(\begin{array}{cc}
\mathbb{I} & \boldsymbol{\xi}_{R}^{T} \\
-\xi_{R}^{*} & \mathbb{I}
\end{array}\right) \simeq\left(\begin{array}{cc}
M_{\chi} & 0 \\
0 & \hat{M}_{\ell}
\end{array}\right)
$$

and the matrices $\boldsymbol{U}_{\boldsymbol{L}}, \boldsymbol{V}_{\boldsymbol{R}}$ diagonalize in turn the chargino mass matrix, with the rotation angles given by

$$
\tan 2 \theta_{L}=-\frac{2 \sqrt{2} M_{W}\left(M_{2} c_{\beta}+\mu s_{\beta}\right)}{M_{2}^{2}-\mu^{2}-2 M_{W}^{2} c_{2 \beta}}, \quad \tan 2 \theta_{R}=-\frac{2 \sqrt{2} M_{W}\left(M_{2} s_{\beta}+\mu c_{\beta}\right)}{M_{2}^{2}-\mu^{2}-2 M_{W}^{2} c_{2 \beta}} .
$$

From equation (31) the matrices $\boldsymbol{\xi}_{\boldsymbol{L}, \boldsymbol{R}}$ are found to be

$$
\boldsymbol{\xi}_{\boldsymbol{R}}^{T}=\boldsymbol{M}_{\chi}^{-1} \boldsymbol{\xi}_{\boldsymbol{L}}^{T} \hat{\boldsymbol{M}}_{\boldsymbol{\ell}} \quad \text { and } \quad \boldsymbol{\xi}_{\boldsymbol{L}}^{*}=\boldsymbol{M}_{\boldsymbol{L} \chi} \boldsymbol{M}_{\chi}^{-1},
$$

which implies $\boldsymbol{\xi}_{\boldsymbol{R}}$ is suppressed with respect $\boldsymbol{\xi}_{\boldsymbol{L}}$ by a factor $m_{\ell} / m_{\text {susy }}$ and thus can be neglected $\left(\boldsymbol{\Xi}_{\boldsymbol{R}}=\mathbb{I}_{5 \times 5}\right)$. Explicitly $\boldsymbol{\xi}_{\boldsymbol{L}}$ can be written in terms of the BRpV parameters and the coefficients entering in the chargino mass matrix:

$$
\begin{aligned}
& \xi_{L_{i 1}}=\frac{g}{\sqrt{2}} \frac{\Lambda_{i}}{\left|\boldsymbol{M}_{\boldsymbol{\chi}}\right|} \text { with } \quad\left|\boldsymbol{M}_{\boldsymbol{\chi}}\right|=M_{2} \mu-M_{W}^{2} s_{2 \beta}, \\
& \xi_{L_{i 2}}=-\frac{2 M_{W}^{2} s_{\beta}}{v\left|\boldsymbol{M}_{\boldsymbol{\chi}}\right| \mu} \Lambda_{i}-\frac{\epsilon_{i}}{\mu}
\end{aligned}
$$

\subsection{CP-even neutral scalars mass matrices and mixings}

In the basis $\left(S^{0}\right)^{T}=\left(\sigma_{d}^{0}, \sigma_{u}^{0}, \tilde{\nu}_{i}^{R}\right)^{T}$ the mass matrix of the CP-even neutral scalars $S^{0}$ is determined by the following quadratic terms

$$
\mathcal{L}_{S}^{0}=\frac{1}{2}\left(S^{0}\right)^{T} \boldsymbol{M}_{\boldsymbol{S}^{0}}^{2} S^{0}=\frac{1}{2}\left(S^{0}\right)^{T}\left(\begin{array}{cc}
\left.\boldsymbol{M}_{\boldsymbol{H} \boldsymbol{H}}^{2}\right|_{2 \times 2} & \left.\boldsymbol{M}_{\boldsymbol{H} \tilde{\boldsymbol{\nu}}}^{2}\right|_{2 \times 3} \\
\left.\boldsymbol{M}_{\boldsymbol{H} \tilde{\boldsymbol{\nu}}}^{2}\right|_{3 \times 2} & \left.\boldsymbol{M}_{\tilde{\boldsymbol{\nu}} \boldsymbol{\nu}}^{2}\right|_{3 \times 3}
\end{array}\right) S^{0} .
$$

In what follows we will discuss approximate analytical formulas for the $\sigma_{u, d}^{0}-\tilde{\nu}_{i}^{R}$ mixing. To our knowledge, this is the first time that they are explicitly given. The entries of the mass matrix in (35) involve the parameters $\mu, \tan \beta$, the soft SUSY breaking coefficients $m_{L_{i}}$ and $B$, and the R-parity breaking parameters $\epsilon_{i}, v_{i}$ and $B_{i}$. We use the minimization conditions of the scalar potential $\left(t_{u, d, i}=0\right)$ to remove the parameters $B_{i}$. In doing so 
the matrix $\boldsymbol{M}_{\boldsymbol{H} \boldsymbol{H}}^{2}$ can be written in terms of the CP-odd neutral scalar mass $m_{A^{0}}$, the sneutrino masses $m_{\tilde{\nu}_{i}}, M_{Z}$ and the BRpV parameters $\epsilon_{i}$ and $v_{i}$, namely

$$
\begin{aligned}
& \left(\boldsymbol{M}_{\boldsymbol{H} \boldsymbol{H}}^{2}\right)_{11}=m_{A^{0}}^{2} s_{\beta}^{2}+M_{Z}^{2} c_{\beta}^{2}+\frac{\mu}{v_{d}} \boldsymbol{\epsilon} \cdot \boldsymbol{v}, \\
& \left(\boldsymbol{M}_{\boldsymbol{H} \boldsymbol{H}}^{2}\right)_{12}=\left(\boldsymbol{M}_{\boldsymbol{H} \boldsymbol{H}}^{2}\right)_{21}=-\left(m_{A^{0}}^{2}+M_{Z}^{2}\right) c_{\beta} s_{\beta}, \\
& \left(\boldsymbol{M}_{\boldsymbol{H} \boldsymbol{H}}^{2}\right)_{22}=m_{A^{0}}^{2} c_{\beta}^{2}+M_{Z}^{2} s_{\beta}^{2}+\sum_{i} \bar{m}_{\tilde{\nu}_{i}}^{2} \frac{v_{i}^{2}}{v_{u}^{2}}-\frac{\mu c_{\beta}^{2}}{v_{d} s_{\beta}^{2}} \sum_{i=1,2,3} \epsilon_{i}^{2} v_{i}^{2}+2 \frac{|\boldsymbol{\epsilon} \cdot \boldsymbol{v}|^{2}}{v_{u}^{2}}+\frac{1}{4} \frac{g_{Z}^{2}}{v_{u}^{2}} \sum_{\substack{i<j \\
j=1,2,3}} v_{i}^{2} v_{j}^{2} .
\end{aligned}
$$

Where the following relations have been used

$$
m_{A^{0}}^{2}=\frac{2 B \mu}{s_{2 \beta}}, \quad \bar{m}_{\tilde{\nu}_{i}}^{2}=m_{\tilde{\nu}_{i}}^{2}+\epsilon_{i}^{2}+\frac{1}{8} g_{Z}^{2} v_{i}^{2} .
$$

Note that phenomenologically consistency requires the inclusion of the one-loop correction in the $\left(\boldsymbol{M}_{\boldsymbol{H} \boldsymbol{H}}^{2}\right)_{22}$ entry, which in the limit of no stop mixing is

$$
\left(\boldsymbol{M}_{\boldsymbol{H} \boldsymbol{H}}^{2}\right)_{22}^{1-\operatorname{loop}}=\frac{3 m_{t}^{4}}{4 \pi^{2} v_{u}^{2}} \log \left(\frac{m_{\tilde{t}_{1}}^{2} m_{\tilde{t}_{2}}^{2}}{m_{t}^{4}}\right) .
$$

See [36] for a more complete expression.

In the absence of BRpV, $\boldsymbol{M}_{\boldsymbol{H} \boldsymbol{H}}^{2}$ corresponds to the neutral CP-even Higgs mass matrix of the R-parity conserving MSSM, which can be diagonalized via the rotation matrix

$$
\boldsymbol{R}_{\alpha}=\left(\begin{array}{cc}
c_{\alpha} & s_{\alpha} \\
-s_{\alpha} & c_{\alpha}
\end{array}\right) \quad \text { with } \quad t_{2 \alpha}=\frac{m_{A}^{2(0)}+M_{Z}^{2}}{m_{A}^{2(0)}-M_{Z}^{2}} t_{2 \beta}
$$

The elements of the $3 \times 3$ right-lower block sneutrino mass matrix in (35) are given by

$$
\begin{aligned}
& \left(\boldsymbol{M}_{\tilde{\boldsymbol{\nu}} \tilde{\boldsymbol{\nu}}}^{2}\right)_{(i+2)(i+2)}=\bar{m}_{\tilde{\nu}_{i}}^{2}+\frac{1}{4} v_{i}^{2}, \\
& \left(\boldsymbol{M}_{\tilde{\boldsymbol{\nu}} \tilde{\boldsymbol{\nu}}}^{2}\right)_{(i+2)(j+2)}=\frac{1}{4} g_{Z}^{2} v_{i} v_{j}+\epsilon_{i} \epsilon_{j} \quad(\text { with } i<j \text { and } i=1,2) .
\end{aligned}
$$

Finally, for the $\sigma_{u, d}^{0}-\tilde{\nu}_{i}^{R}$ mixing $2 \times 3$ block we have

$$
\begin{aligned}
& \left(\boldsymbol{M}_{\boldsymbol{H} \tilde{\boldsymbol{\nu}})_{1(i+2)}}^{2}=-\mu^{2} \frac{\epsilon_{i}}{\mu}+M_{Z}^{2} c_{\beta}^{2} \frac{v_{i}}{v_{d}}\right. \\
& \left(\boldsymbol{M}_{\boldsymbol{H} \tilde{\boldsymbol{\nu}})_{2(i+2)}}^{2}=\frac{\mu^{2}}{t_{\beta}} \frac{\epsilon_{i}}{\mu}-\left(\bar{m}_{\tilde{\nu}_{i}}^{2}+M_{Z}^{2} s_{\beta}^{2}\right) \frac{1}{t_{\beta}} \frac{v_{i}}{v_{d}}-\frac{1}{2} M_{Z}^{2} c_{\beta}^{3} \frac{v_{i}}{v_{d}} \sum_{j \neq i} \frac{v_{j}^{2}}{v_{d}^{2}}-\frac{\mu^{2}}{t_{\beta}} \frac{\epsilon_{i}}{\mu} \sum_{i \neq j} \frac{v_{j}}{v_{d}} \frac{\epsilon_{j}}{\mu} .\right.
\end{aligned}
$$

In the mass eigenstate basis defined as

$$
S^{\prime 0}=\boldsymbol{R}^{S^{0}} S^{0},
$$


the Lagrangian in (35) becomes

$$
\mathcal{L}_{S^{0}}=\frac{1}{2}\left(S^{\prime 0}\right)^{T} \hat{\boldsymbol{M}}_{\boldsymbol{S}^{\mathbf{0}}}^{\mathbf{2}} S^{\prime 0} \quad \text { with } \quad \hat{\boldsymbol{M}}_{\boldsymbol{S}^{\mathbf{0}}}^{2}=\boldsymbol{R}^{\boldsymbol{S}^{\mathbf{0}}} \boldsymbol{M}_{\boldsymbol{S}^{\mathbf{0}}}{ }^{2} \boldsymbol{R}^{\boldsymbol{S}^{\mathbf{0}}}
$$

where $\hat{\boldsymbol{M}}_{\boldsymbol{S}^{\mathbf{0}}}^{2}$ is diagonal. Assuming real parameters $\boldsymbol{R}^{\boldsymbol{S}^{\mathbf{0}}}$ can be parameterized as

$$
\left(\boldsymbol{R}^{\boldsymbol{S}^{\mathbf{0}}}\right)^{T}=\prod_{\substack{i<j \\ j=1, \cdots, 5}}\left(\boldsymbol{R}_{i j}\right)^{T}
$$

where the $\boldsymbol{R}_{i j} \equiv \boldsymbol{R}\left(\theta_{i j}\right)$ are $5 \times 5$ rotation matrices.

If the $\sigma_{d, u}^{0}-\tilde{\nu}_{i}^{R}$ mixing is small - as expected due to the smallness of the BRpV parameters required by neutrino data - a perturbative diagonalization of the mass matrix in (35) can be done. By neglecting the BRpV parameters in $\boldsymbol{M}_{\boldsymbol{H} \boldsymbol{H}}^{2}$ and $\boldsymbol{M}_{\tilde{\boldsymbol{\nu}} \tilde{\boldsymbol{\nu}}}^{2}$ the rotation matrix reduces to

$$
R^{S^{0}}=R_{25} R_{24} R_{23} R_{15} R_{14} R_{13} R_{12}
$$

When acting on $\boldsymbol{M}_{\boldsymbol{S}^{\mathbf{0}}}^{2}$ the matrix $\boldsymbol{R}_{\mathbf{1 2}}$ diagonalizes the $2 \times 2$ block $\boldsymbol{M}_{\boldsymbol{H} \boldsymbol{H}}^{2}$ according to $\hat{\boldsymbol{M}}_{\boldsymbol{H} \boldsymbol{H}}^{2}=\operatorname{diag}\left(m_{H^{0}}^{2}, m_{h^{0}}^{2}\right)$ (where $h^{0}$ and $H^{0}$ are the light and heavy CP-even Higgs bosons) and modifies the BRpV mixing matrix $\boldsymbol{M}_{\boldsymbol{H} \tilde{\boldsymbol{\nu}}}^{2}$ :

$$
\boldsymbol{M}_{\boldsymbol{H} \tilde{\boldsymbol{\nu}}}^{2} \rightarrow\left(\begin{array}{c}
s_{\alpha}\left(\boldsymbol{M}_{\boldsymbol{H} \tilde{\boldsymbol{\nu}}}^{2}\right)_{2(i+2)}+c_{\alpha}\left(\boldsymbol{M}_{\boldsymbol{H} \tilde{\boldsymbol{\nu}}}^{2}\right)_{1(i+2)} \\
c_{\alpha}\left(\boldsymbol{M}_{\boldsymbol{H} \tilde{\boldsymbol{\nu}}}^{2}\right)_{2(i+2)}-s_{\alpha}\left(\boldsymbol{M}_{\boldsymbol{H} \tilde{\boldsymbol{\nu}}}^{2}\right)_{1(i+2)}
\end{array}\right)
$$

For the mixing angle we have $\theta_{12}=\alpha$. The matrices $\boldsymbol{R}_{\mathbf{1}(\boldsymbol{i}+\mathbf{2})}$ eliminate the first row entries

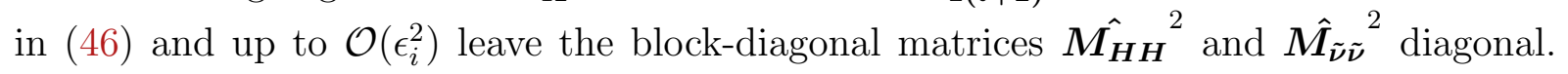
The rotation angles are given by

$$
\theta_{1(i+2)} \simeq \frac{s_{\alpha}\left(\boldsymbol{M}_{\boldsymbol{H} \tilde{\boldsymbol{\nu}}}^{2}\right)_{2(i+2)}+c_{\alpha}\left(\boldsymbol{M}_{\boldsymbol{H} \tilde{\boldsymbol{\nu}}}^{2}\right)_{1(i+2)}}{m_{H^{0}}^{2}-m_{\tilde{\nu}_{i}}^{2}} .
$$

The matrices $\boldsymbol{R}_{\mathbf{2}(\boldsymbol{i + 2})}$ instead eliminate the second row elements in (46) leaving again, up to order $\epsilon_{i}^{2}$, the block-diagonal matrices diagonal. The rotation angles in this case read

$$
\theta_{2(i+2)} \simeq \frac{c_{\alpha}\left(\boldsymbol{M}_{\boldsymbol{H} \tilde{\boldsymbol{\nu}}^{2}}\right)_{2(i+2)}-s_{\alpha}\left(\boldsymbol{M}_{\boldsymbol{H} \tilde{\boldsymbol{\nu}}}^{2}\right)_{1(i+2)}}{m_{h^{0}}^{2}-m_{\tilde{\nu}_{i}}^{2}}
$$

With these results at hand and neglecting terms of $\mathcal{O}\left(\theta^{2}\right)$ the full rotation matrix in (45) can be written as

$$
\boldsymbol{R}^{S^{0}} \simeq\left(\begin{array}{cc}
\boldsymbol{R}_{\alpha} & \boldsymbol{R}_{\tilde{\nu}} \\
\boldsymbol{R}_{\sigma} & \mathbb{I}
\end{array}\right)
$$

where $\boldsymbol{R}_{\tilde{\boldsymbol{\nu}}}$ and $\boldsymbol{R}_{\boldsymbol{\sigma}}$ account for the sneutrino and CP-even neutral Higgs components of the mass eigenstates, and read

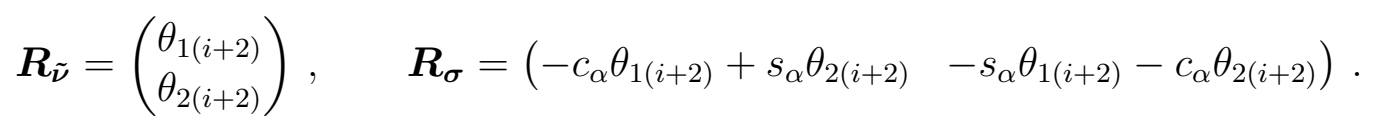




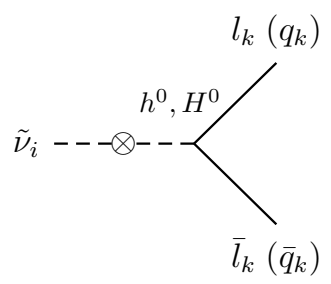

(a)

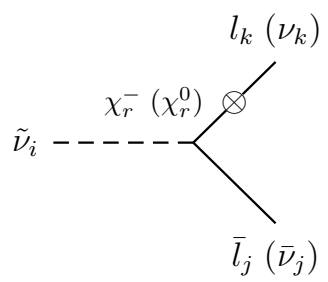

(b)

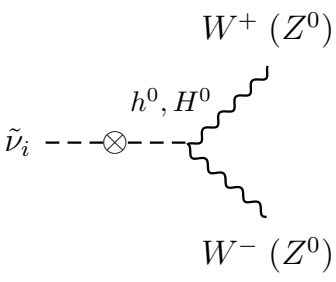

$(c)$

Figure 1: BRpV induced sneutrino decay modes. In $(a)$ and $(c)$ sneutrino decays are induced by sneutrino-Higgs mixing while in figure (b) by chargino-charged lepton or neutralino-neutrino mixing, depending on whether the final states involves charged lepton or neutrinos. The open circles with a cross inside indicate a BRpV mixing insertion.

\section{Sneutrino decays}

With the results of section 3 we are now in a position to discuss approximate formulas for sneutrino decays. From now on we will consider only CP-even sneutrino decays, $\tilde{\nu}^{R}$, and so will drop the superscript $R$. Possible tree-level two-body sneutrino final states include fermionic modes $\tilde{\nu}_{i} \rightarrow l_{j}^{+} l_{k}^{-}, \nu_{j} \nu_{k}$ and $q_{k} \bar{q}_{k}$; electroweak gauge bosons modes $\tilde{\nu}_{i} \rightarrow W^{+} W^{-}$ and $Z^{0} Z^{0}$ and Higgs bosons modes $\tilde{\nu}_{i} \rightarrow h^{0} h^{0}, H^{0} H^{0}, A^{0} A^{0}$ and $H^{+} H^{-}$.

The goal of this paper and section is not a full study of the parameter space and all decays but rather to show that these heavy states can dominate the traditionally considered decay to $b \bar{b}$ and to identify the relevant parameter space for this dominance.

\subsection{Relevant Lagrangians}

Taking into account our approximate results for the chargino-right-handed lepton mixing, $\xi_{R} \sim\left(m_{\ell} / m_{\text {susy }}\right)$, mixing with right-handed leptons is zero $\left(\xi_{R} \sim 0\right.$, see eq. (33)), the full Lagrangian for sneutrino decays into charged leptons is reduced to [32]

$$
-\mathcal{L}^{( \pm)}=\frac{h_{k}^{E}}{\sqrt{2}}\left(\boldsymbol{R}^{S^{\mathbf{0}}}\right)_{(i+2) 1} \overline{l_{k}^{-}} P_{L} l_{k}^{-} \tilde{\nu}_{i}-\frac{h_{i}^{E}}{\sqrt{2}} \boldsymbol{U}_{(j+2) 2} \overline{l_{i}^{-}} P_{L} l_{j}^{-} \tilde{\nu}_{i}+\text { H.c. }
$$

where the mixing matrices are given by eqs. (34) and (49). Due to the smallness of the BRpV induced mixing we take $S_{(i+2)}^{\prime 0} \rightarrow \tilde{\nu}_{i}$. Note that while the first term in (51) necessarily leads to two same-flavor opposite-sign charged lepton final states the second term can lead to different-flavor signatures. The corresponding Feynman diagrams for these processes are depicted in figure $1((a),(b))$. Being proportional to SM charged lepton Yukawa couplings these decays are dominated by final states involving $\tau$ 's.

For sneutrino decays into neutrinos (invisible decays $\tilde{\nu}_{i} \rightarrow \sum_{k, j} \nu_{k} \nu_{j}$ ), the relevant interactions are given by

$$
-\mathcal{L}^{(0)}=\frac{1}{2} \overline{\nu_{k}}\left(C_{k j i}+C_{j k i}\right) \nu_{j} \tilde{\nu}_{i}+\text { H.c. },
$$




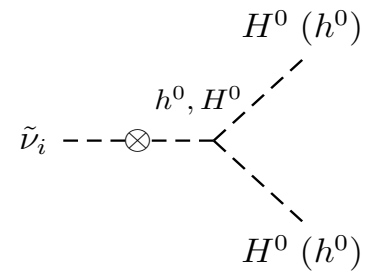

(a)

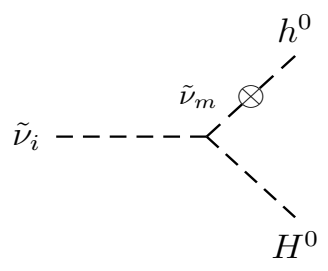

(b)

Figure 2: BRpV induced sneutrino decays involving Higgs final states. The open circles with a cross inside indicate a BRpV mixing insertion.

where the coupling reads

$$
C_{k j i}=\bar{N}_{k+4} \sum_{n=1}^{5} s_{n} \boldsymbol{N}_{(j+4)(n+2)}\left(\boldsymbol{R}^{S^{\mathbf{0}}}\right)_{(i+2) n}
$$

where $s_{n}=(1,-1,1,1,1)$ and

$$
\bar{N}_{k+4}=g^{\prime} \boldsymbol{N}_{(k+4) 1}-g \boldsymbol{N}_{(k+4) 2} .
$$

The elements of the neutral fermion mixing matrix correspond to the entries of the matrix given in eq. (16). These interactions induce invisible sneutrino decays as the ones shown in figure $1(b)$.

In the up and down quark mass eigenstate basis the sneutrino-quark-quark interactions are dictated by

$$
\mathcal{L}^{(q)}=-\frac{1}{\sqrt{2}} h_{k}^{U}\left(\boldsymbol{R}^{\boldsymbol{S}^{\mathbf{0}}}\right)_{(i+2) 2} \bar{u}_{k} u_{k} \tilde{\nu}_{i}-\frac{1}{\sqrt{2}} h_{k}^{D}\left(\boldsymbol{R}^{\boldsymbol{S}^{\mathbf{0}}}\right)_{(i+2) 1} \bar{d}_{k} d_{k} \tilde{\nu}_{i} .
$$

As for the charged lepton final states, these decays are controlled by SM quark Yukawa couplings and thus are dominated by $b \bar{b}$ and $t \bar{t}$, the last one if kinematically allowed.

The Lagrangian for gauge boson final states is given by

$$
\mathcal{L}^{(V)}=g_{V}\left(c_{\beta}\left(\boldsymbol{R}^{\boldsymbol{S}^{\mathbf{0}}}\right)_{(i+2) 1}+s_{\beta}\left(\boldsymbol{R}^{\boldsymbol{S}^{\mathbf{0}}}\right)_{(i+2) 2}+\frac{v_{i}}{v}\right) V^{\mu} V_{\mu} \tilde{\nu}_{i}
$$

with $V_{\mu}=W_{\mu}, Z_{\mu}$ and $g_{W, Z}=g M_{W}, g M_{Z} / c_{W}$. For Higgs final states we write the Lagrangian involving $h^{0}$ and $H^{08}$ :

$$
\mathcal{L}^{S^{0}}=-g_{i j k} S_{j}^{0} S_{k}^{0} \tilde{\nu}_{i},
$$

where the fully symmetric coupling $g_{(i+2) j k}$ is given by

$$
g_{i j k}=-\frac{1}{4} g_{Z}^{2} \sum_{n=1}^{5} u_{n}\left[\left(\boldsymbol{R}^{\boldsymbol{S}^{\mathbf{0}}}\right)_{(i+2) n} \bar{R}_{j k}+\left(\boldsymbol{R}^{\left.\boldsymbol{S}^{\mathbf{0}}\right)_{j n}} \bar{R}_{(i+2) k}+\left(\boldsymbol{R}^{\boldsymbol{S}^{\mathbf{0}}}\right)_{k n} \bar{R}_{(i+2) j}\right]\right.
$$

\footnotetext{
${ }^{8}$ We do not study $\tilde{\nu}_{i} \rightarrow A^{0} A^{0}$ and $\tilde{\nu}_{i} \rightarrow H^{+} H^{-}$decays and so do not present the Lagrangians that govern these interactions.
} 
with $u_{n}=\left(u_{1}, u_{2}, u_{3} \ldots\right)=\left(v_{d},-v_{u}, v_{1} \ldots\right)$ and

$$
\bar{R}_{j k}=\left(\boldsymbol{R}^{S^{\mathbf{0}}}\right)_{j 1}\left(\boldsymbol{R}^{S^{\mathbf{0}}}\right)_{k 1}-\left(\boldsymbol{R}^{S^{\mathbf{0}}}\right)_{j 2}\left(\boldsymbol{R}^{S^{\mathbf{0}}}\right)_{k 2}+\sum_{n=3}^{5}\left(\boldsymbol{R}^{S^{\mathbf{0}}}\right)_{j n}\left(\boldsymbol{R}^{\boldsymbol{S}^{\mathbf{0}}}\right)_{k n} .
$$

The interactions in (57) for $j=k=1,2$ lead to decays of type $(a)$ in figure 2 while for $j=1, k=2$ to those shown in figure $2(b)$.

\subsection{Partial decay widths}

Fermionic final states are dominated by third generation quark and charged leptons. Due to the structure of the Higgs-sneutrino mixing, $\tau \bar{\tau}, b \bar{b}$ and $t \bar{t}$ final states are possible independently of the sneutrino flavor, whereas $\bar{\tau}(e, \mu)$ final states are only sizable for tau and mu sneutrinos. Neglecting the final state masses, the partial decay widths for $\bar{\tau}(e, \mu)$, $\tau \bar{\tau}$ and $b \bar{b}$ decays can be written as

$$
\begin{aligned}
\Gamma\left(\tilde{\nu}_{i} \rightarrow \bar{l}_{i} l_{k}\right) & =\frac{m_{l_{i}}^{2} G_{F}}{4 \sqrt{2} \pi c_{\beta}^{2}} m_{\tilde{\nu}_{i}} \boldsymbol{U}_{(k+2) 2}^{2}, \\
\Gamma\left(\tilde{\nu}_{i} \rightarrow \tau \bar{\tau}\right) & =\frac{m_{\tau}^{2} G_{F}}{4 \sqrt{2} \pi c_{\beta}^{2}} m_{\tilde{\nu}_{i}}\left[\left(\boldsymbol{R}^{S^{0}}\right)_{(i+2) 1}-\boldsymbol{U}_{52} \delta_{i 3}\right]^{2}, \\
\Gamma\left(\tilde{\nu}_{i} \rightarrow b \bar{b}\right) & =\frac{3 m_{b}^{2} G_{F}}{4 \sqrt{2} \pi c_{\beta}^{2}} m_{\tilde{\nu}_{i}}\left(\boldsymbol{R}^{S^{0}}\right)_{(i+2) 1}^{2},
\end{aligned}
$$

where $G_{F}$ is the Fermi constant. For invisible decay modes the partial decay width, summing over lepton flavors, can be written according to ${ }^{9}$

$$
\Gamma\left(\tilde{\nu}_{i} \rightarrow \sum_{k, j} \nu_{k} \nu_{j}\right)=\frac{m_{\tilde{\nu}_{i}}}{16 \pi} \sum_{k, j}\left(C_{k j i}+C_{j k i}\right)^{2},
$$

with $C_{k j i}$ given by (53). For $t \bar{t}$ final states the phase space factors are relevant, accordingly the corresponding decay width reads

$$
\Gamma\left(\tilde{\nu}_{i} \rightarrow t \bar{t}\right)=\frac{3 m_{t}^{2} G_{F}}{4 \sqrt{2} \pi s_{\beta}^{2}} m_{\tilde{\nu}_{i}}\left(\boldsymbol{R}^{S^{0}}\right)_{(i+2) 2}^{2}\left(1-4 \frac{m_{t}^{2}}{m_{\tilde{\nu}_{i}}^{2}}\right)^{3 / 2}
$$

For Gauge boson final states the partial decay width is given by

$$
\Gamma\left(\tilde{\nu}_{i} \rightarrow V V\right)=\frac{G_{F} m_{\tilde{\nu}_{i}}^{3}}{16 \sqrt{2} \pi} \delta_{V} \sqrt{1-4 \frac{M_{V}^{2}}{m_{\tilde{\nu}_{i}}^{2}}}\left(1-4 \frac{M_{V}^{2}}{m_{\tilde{\nu}_{i}}^{2}}+12 \frac{M_{V}^{4}}{m_{\tilde{\nu}_{i}}^{4}}\right)\left|\mathcal{A}_{i}^{V}\right|^{2},
$$

where $V=Z, W, \delta_{Z, W}=1,2$ and the amplitude $A_{i}^{V}$ reads

$$
\mathcal{A}_{i}^{V}=c_{\beta}\left(\boldsymbol{R}^{S^{0}}\right)_{(i+2) 1}+s_{\beta}\left(\boldsymbol{R}^{S^{0}}\right)_{(i+2) 2}+\frac{v_{i}}{v} .
$$

\footnotetext{
${ }^{9}$ These modes were studied in ref. [37]
} 
For $V V^{*}$ final states the partial decay width is given by [38]

$$
\frac{d \Gamma\left(\tilde{\nu}_{i} \rightarrow V V^{*}\right)}{d x_{1} d x_{2}}=K_{\tilde{\nu}_{i} V V} \frac{\left(1-x_{1}\right)\left(1-x_{2}\right)+\kappa_{V}\left(2 x_{1}+2 x_{2}-3+2 \kappa_{V}\right)}{\left(1-x_{1}-x_{2}\right)^{2}+\kappa_{V} \gamma_{V}},
$$

with

$$
\kappa_{V}=\frac{M_{V}^{2}}{m_{\tilde{\nu}_{i}}^{2}}, \quad K_{\tilde{\nu}_{i} V V}=\frac{3 G_{F}^{2} M_{W}^{4}}{16 \pi^{3}}\left|\mathcal{A}_{V}^{i}\right|^{2} m_{\tilde{\nu}_{i}} 3 \delta_{V}^{\prime}
$$

and

$$
\delta_{W}^{\prime}=1, \quad \delta_{Z}^{\prime}=\frac{1}{c_{W}^{4}}\left(\frac{7}{12}-\frac{10}{9} s_{W}^{2}+\frac{40}{9} s_{W}^{4}\right) .
$$

The integration variables $x_{1,2}$ lie in the ranges $x_{1}=\left[1-x_{2}-\kappa_{V}, 1-\kappa_{V} /\left(1-x_{2}\right)\right]$ and $x_{2}=\left[0,1-\kappa_{V}\right]$. The parameter $\gamma_{V}=\Gamma_{V}^{2} / M_{V}^{2}$ ( $\Gamma_{V}$ being the total decay width of the gauge boson $V$ ) allows a smooth transition in the threshold region where the off-shell gauge boson becomes on-shell $\left(m_{\tilde{\nu}_{i}}=2 M_{V}\right)$, the calculation of $\Gamma\left(\tilde{\nu}_{i} \rightarrow V V^{*}\right)$ thus requires numerical integration over the variables $x_{1,2}$ in the transition region. Outside that region, i.e. for $m_{\tilde{\nu}_{i}} \lesssim 2 M_{V}-\Gamma_{V}$ it can be written according to [39]

$$
\Gamma\left(\tilde{\nu}_{i} \rightarrow V V^{*}\right)=\frac{3 G_{F}^{2} M_{V}^{4}}{16 \sqrt{2} \pi} m_{\tilde{\nu}_{i}} \delta_{V}^{\prime} R_{T}\left(\frac{M_{V}^{2}}{m_{\tilde{\nu}_{i}}^{2}}\right)\left|\mathcal{A}_{i}^{V}\right|^{2},
$$

where $R_{T}(x)$ is a kinematic function given by

$$
\begin{aligned}
R_{T}(x) & =\frac{3\left(1-8 x+20 x^{2}\right)}{\sqrt{4 x-1}} \arccos \left(\frac{3 x-1}{2 x^{3 / 2}}\right)-\frac{1-x}{2 x}\left(2-13 x+47 x^{2}\right) \\
& -\frac{3}{2}\left(1-6 x+4 x^{2}\right) \log x .
\end{aligned}
$$

Finally for CP-even Higgs bosons final states the partial decay width can be written as

$$
\Gamma\left(\tilde{\nu}_{i} \rightarrow S_{j}^{0} S_{k}^{0}\right)=\frac{g_{(i+2) j k}^{2}}{16 \pi m_{\tilde{\nu}_{i}}^{3}} \lambda^{1 / 2}\left(m_{\tilde{\nu}_{i}}^{2}, m_{S_{j}^{0}}^{2}, m_{S_{k}^{0}}^{2}\right) \quad(j, k=1,2),
$$

where $\lambda(a, b, c)=(a-b-c)^{2}-4 b c, S_{1,2}^{0}=H^{0}, h^{0}$ and the dimensionful coupling $g_{(i+2) j k}$ is given in (58). For the particular case $j=k\left(h^{0} h^{0}\right.$ and $H^{0} H^{0}$ final states) the width reduces to

$$
\Gamma\left(\tilde{\nu}_{i} \rightarrow S_{j}^{0} S_{j}^{0}\right)=\frac{g_{i j j}^{2}}{32 \pi m_{\tilde{\nu}_{i}}} \sqrt{1-4 \frac{m_{S_{j}^{0}}^{2}}{m_{\tilde{\nu}_{i}}^{2}}}
$$

\subsection{Constraints on $\mathrm{BRpV}$ parameters from neutrino data}

Neutrino data plays an important role in $\mathrm{BRpV}$, since $\mathrm{BRpV}$ parameters contribute to neutrino masses. Therefore, even if $\mathrm{BRpV}$ is not solely responsible for neutrino masses, 

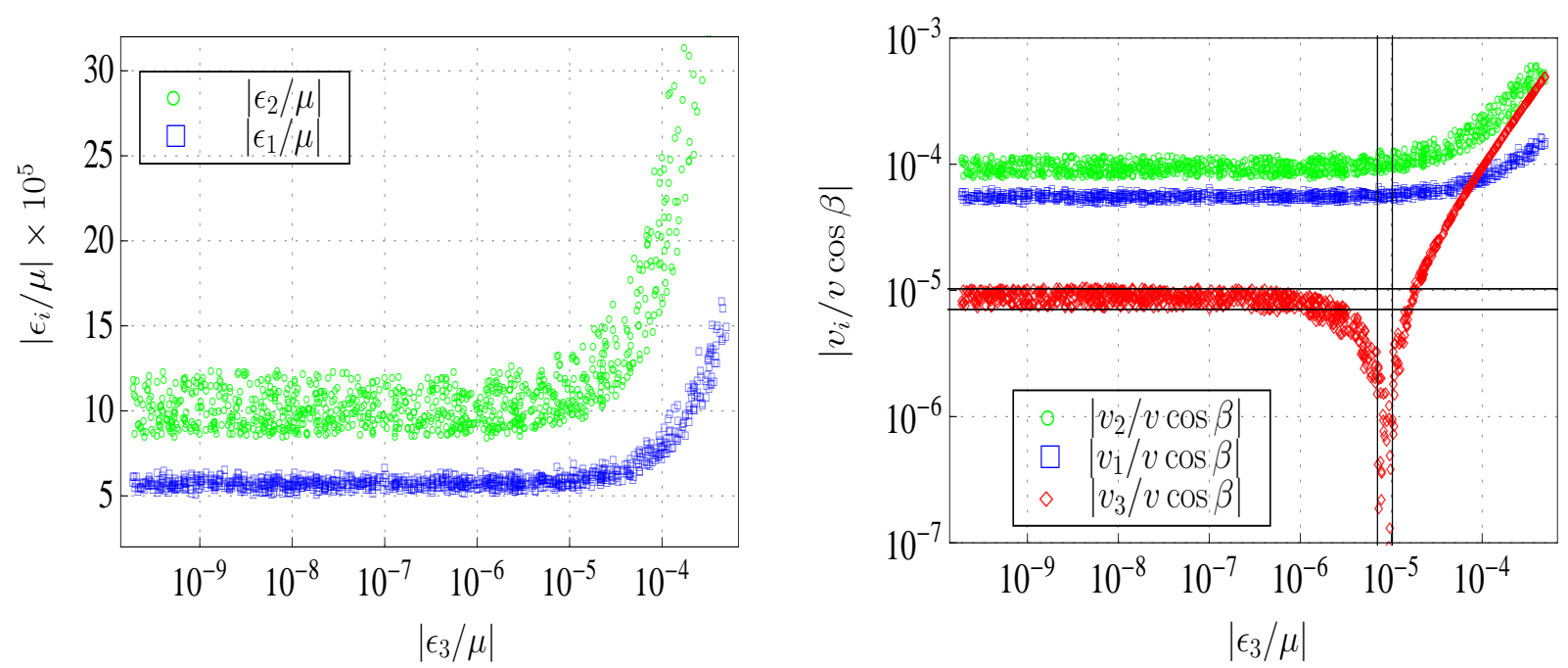

Figure 3: Numerical ranges for $\epsilon_{i}(i=1,2,3)$ parameters and sneutrino vevs as required for explaining neutrino data for the R-parity conserving parameters discussed in the text. The horizontal (vertical) solid lines in the right-panel plot indicate the values where $\Lambda_{3}$ is dominated by $v_{3}\left(\epsilon_{3}\right)$, i.e. where $\Lambda_{3} \simeq \mu v_{3}\left(\Lambda_{3} \simeq v \cos \beta \epsilon_{3}\right)$.

e.g. [11, 21, 41, 42, 43], one must not saturate neutrino masses via BRpV. Therefore it would be worthwhile to discuss this correlation briefly here. We proceed by assuming $\mathrm{BRpV}$ as the sole source of neutrino masses and later consider relaxing this assumption.

At tree-level, BRpV allows for only one massive neutrino, as mentioned earlier. Oneloop contributions then leave only one generation massless. Furthermore, for the approximations made in this paper, the tree-level mass is larger than the one-loop mass. The upshot of all this is that only the so-called normal hierarchy of neutrino masses is allowed. Recent neutrino data $[44,45,46]$ then specifies the following neutrino masses:

$$
m_{1}=0, \quad m_{2}=\sqrt{\Delta m_{21}^{2}}=0.00873 \mathrm{eV}, \quad m_{3}=\sqrt{\Delta m_{31}^{2}}=0.0505 \mathrm{eV} .
$$

In terms of the model parameters

$$
\Delta m_{32}^{\mathrm{BRpV}}=\frac{M_{\tilde{\gamma}}}{4\left|\boldsymbol{M}_{\boldsymbol{\chi}}^{\mathbf{0}}\right|}|\boldsymbol{\Lambda}|^{2} .
$$

The experimental values of the atmospheric and reactor angles yield two additional constraints given by eq. (21). Therefore, the atmospheric sector entirely fixes the $\Lambda_{i}$ parameters.

Constraints on the $\epsilon$ parameters arise from the solar sector. As long as the one-loop contribution to neutrino masses is smaller than the tree-level one (an assumption we use throughout), $\Delta m_{21}^{\mathrm{BRpV}}$ is accurately determined by $b-\tilde{b}$ one-loop diagrams [33]:

$$
\Delta m_{21}^{\mathrm{BRpV}}=\frac{3}{8 \pi^{2}} \sin 2 \theta_{\tilde{b}} \frac{m_{b}^{3}}{v^{2} c_{\beta}^{2}} \Delta B_{0}^{\tilde{b}_{2} \tilde{b}_{1}} \frac{\left(\bar{\epsilon}_{1}^{2}+\bar{\epsilon}_{2}^{2}\right)}{\mu^{2}},
$$


where $\theta_{\tilde{b}}$ stands for sbottom mixing, $\bar{\epsilon}_{1,2}$ are defined in eq. (24) and $\Delta B_{0}^{\tilde{b}_{2} \tilde{b}_{1}}=B_{0}\left(0, m_{\tilde{b}_{2}}^{2}, m_{b}^{2}\right)-$ $B_{0}\left(0, m_{\tilde{b}_{1}}^{2}, m_{b}^{2}\right)$ (with $B_{0}(0, x, y)$ is a scalar Passarino-Veltman function [47]). Note that due to $m_{b} \ll m_{\tilde{b}_{1,2}}$,

$$
\Delta B_{0}^{\tilde{b}_{2} \tilde{b}_{1}} \simeq \log \left(\frac{m_{\tilde{b}_{2}}^{2}}{m_{\tilde{b}_{1}}^{2}}\right) .
$$

Moreover the solar mixing angle can be written as [33]

$$
\tan ^{2} \theta_{12}^{\mathrm{BRpV}}=\frac{\bar{\epsilon}_{1}^{2}}{\bar{\epsilon}_{2}^{2}} .
$$

Thus, eqs. (76) and (78) provide two constraints and determine, from eqs. $(24), \epsilon_{1,2}\left(\epsilon_{1,3}\right.$ or $\left.\epsilon_{2,3}\right)$ as a function of $\epsilon_{3}\left(\epsilon_{2}\right.$ or $\left.\epsilon_{1}\right)$. Once the $\Lambda$ 's and $\epsilon$ 's are fixed the sneutrino vevs are automatically fixed as well through

$$
v_{i}=\frac{\Lambda_{i}-v_{d} \epsilon_{i}}{\mu}
$$

see eq. (18). This in turn fixes all the relevant mixings for sneutrino decays $\left(\chi^{0}-\nu, \chi^{-}-\ell_{L}^{-}\right.$, $\left.\sigma_{d, u}^{0}-\tilde{\nu}_{i}^{R}\right)$ once the R-parity conserving supersymmetric parameters are specified. With this knowledge in hand, we start to explore the consequences of the neutrino sector on the BRpV parameters. We note that our results have been verified using SPheno [48] $]^{10}$.

Figure 3 shows typical values for $\epsilon_{1,2}$ and sneutrino vevs as a function of $\epsilon_{3}$. The plots were obtained by fixing $\theta_{\tilde{b}}=\pi / 16, \tan \beta=10$ and

$$
\left(\mu, M_{1}, M_{2}, m_{\tilde{b}_{2}}, m_{\tilde{b}_{1}}, m_{A}, m_{\tilde{\nu}_{i}}\right)=\left(650,550,600,10^{3}, 700,10^{3}, 300\right) \mathrm{GeV},
$$

where we have assumed a conservative lower bound on the sneutrino mass of $100 \mathrm{GeV}$, see [49] for more details. We will use this parameter point throughout the paper. Neutrino observable $\left(\Delta m_{32}, \Delta m_{21}, \theta_{i j}\right)$ are varied in their $3 \sigma$ experimental range $[44,45,46]$. We further assume that $\epsilon_{3}$ is positive. For these assumptions we see from the left-hand side that $\epsilon_{1}$ and $\epsilon_{2}$ have a lower bound and from the right-handed side that this lower bound is larger than $v_{1}$ and $v_{2}$ respectively. On the other hand, since $\epsilon_{3}$ is undetermined, $v_{3}$ dominates it up to about $\left|\epsilon_{3} / \mu\right|=10^{-5}$. While allowing negative values for the parameters changes this picture, it leaves one important qualitative property the same: only in one generation, $j$, can the sneutrino vev, $v_{j}$, be larger than the bilinear mixing term, $\epsilon_{j}$, when BRpV is the sole contributor to neutrino masses. This will have important consequences in the next section.

Also of note in the left-panel plot, $\epsilon_{3}$ obeys only an upper bound determined by

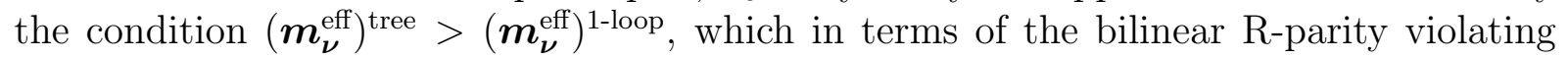
parameters translates into $|\boldsymbol{\Lambda}|>|\boldsymbol{\epsilon}|^{2}$. In contrast the $\epsilon_{1,2}$ parameters, due to solar neutrino physics constraints, are forced to lie in a "narrow" range and are such that a region where

\footnotetext{
${ }^{10}$ We thank Werner Porod for fixing a bug in the decay routines which allowed us to calculate sneutrino decays to gauge bosons, discussed in section 4.4
} 


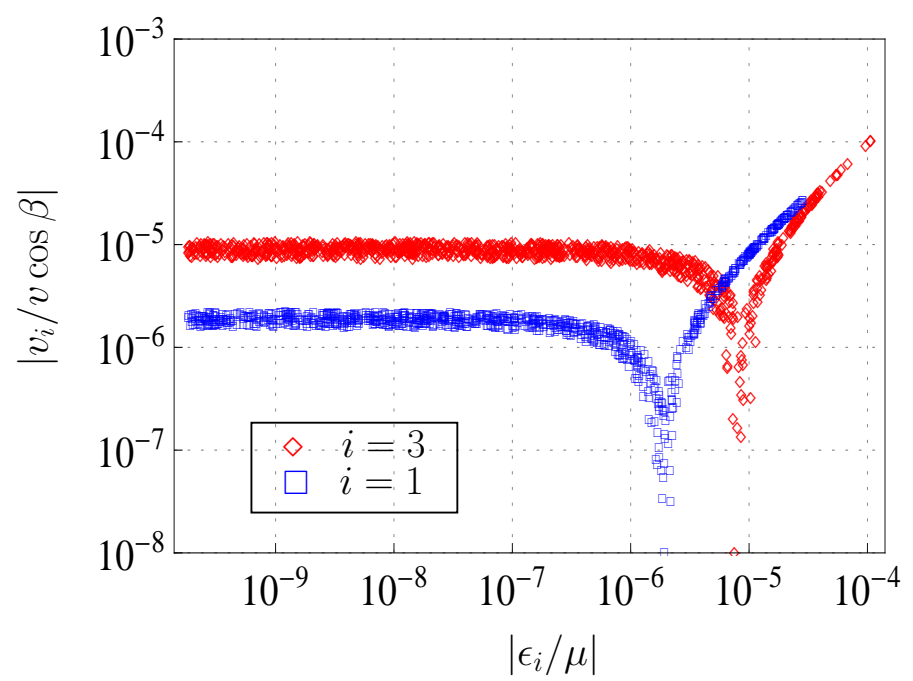

Figure 4: Values of R-parity breaking parameters for which BRpV is not the sole source of neutrino physics. The parameters are such that $\Delta m_{32}^{B R p V}$ fits the experimental $3 \sigma$ range but $\Delta m_{21}^{B R P V}$ falls below the measured value (see the text for more details).

$\epsilon_{1,2} \gg \epsilon_{3}$ exist. Consequently, while $\Lambda_{1,2}$ are mostly determined by $\epsilon_{1,2}, \Lambda_{3}$ is controlled by $v_{3}$ in the region where $\epsilon_{3} / \mu \lesssim 10^{-7}$, as demonstrated by the horizontal solid lines in figure 3 (right-panel) which correspond to $\Lambda_{3} \simeq \mu v_{3}$.

The bilinear R-parity breaking parameters selected as described above satisfy neutrino data, and thus lead to BRpV models that can account for neutrino masses and mixings. However, it might be that these parameters are not sufficiently large to account for the neutrino mass scales. In that case their contribution to the atmospheric and solar masses are still determined by eqs. (75) and (76) but are such that, for example, $\Delta m_{31}^{\mathrm{BRpV}}<(\ll) \Delta m_{31}^{\mathrm{Exp}}$ and $\Delta m_{21}^{\mathrm{BRpV}}<(\ll) \Delta m_{21}^{\text {Exp }}$. Figure 4 shows the results for an illustrative case where the $\mathrm{BRpV}$ model fits the atmospheric mass scale as well as the atmospheric and reactor angles in their $3 \sigma$ experimental range, but the contribution to $\Delta m_{21}$ is subdominant ${ }^{11}$. Note that we plot only $v_{1,3}$, as we have found that $v_{2} \sim v_{3}$. This result as well as $v_{1} \ll v_{3}$ in the region of small $\epsilon_{1,3}$ are due to the constraints arising from fitting $\theta_{23}$ and $\theta_{13}$.

In summary, if data is not explained by the $\mathrm{BRpV}$ parameters all the $\epsilon$ 's can be small mainly due to the absence of the solar data constraint, thus implying that, in this case, a region where the three sneutrino vevs are large $\left(\Lambda_{i} \simeq \mu v_{i}\right)$ exist.

\subsection{LSP sneutrino phenomenology}

We are finally ready to address the main aim of this paper: showing that sneutrino LSP decays into heavy SM pairs, invisible modes and different lepton flavor final states i.e. $W^{+} W^{-}, Z Z, h^{0} h^{0}, t \bar{t} ; \nu \nu$ and $l_{i}^{+} l_{k}^{-}(i \neq k)$ can dominate over the traditionally considered

\footnotetext{
${ }^{11}$ Models of this kind have been considered in the literature, see e.g. [40, 41].
} 

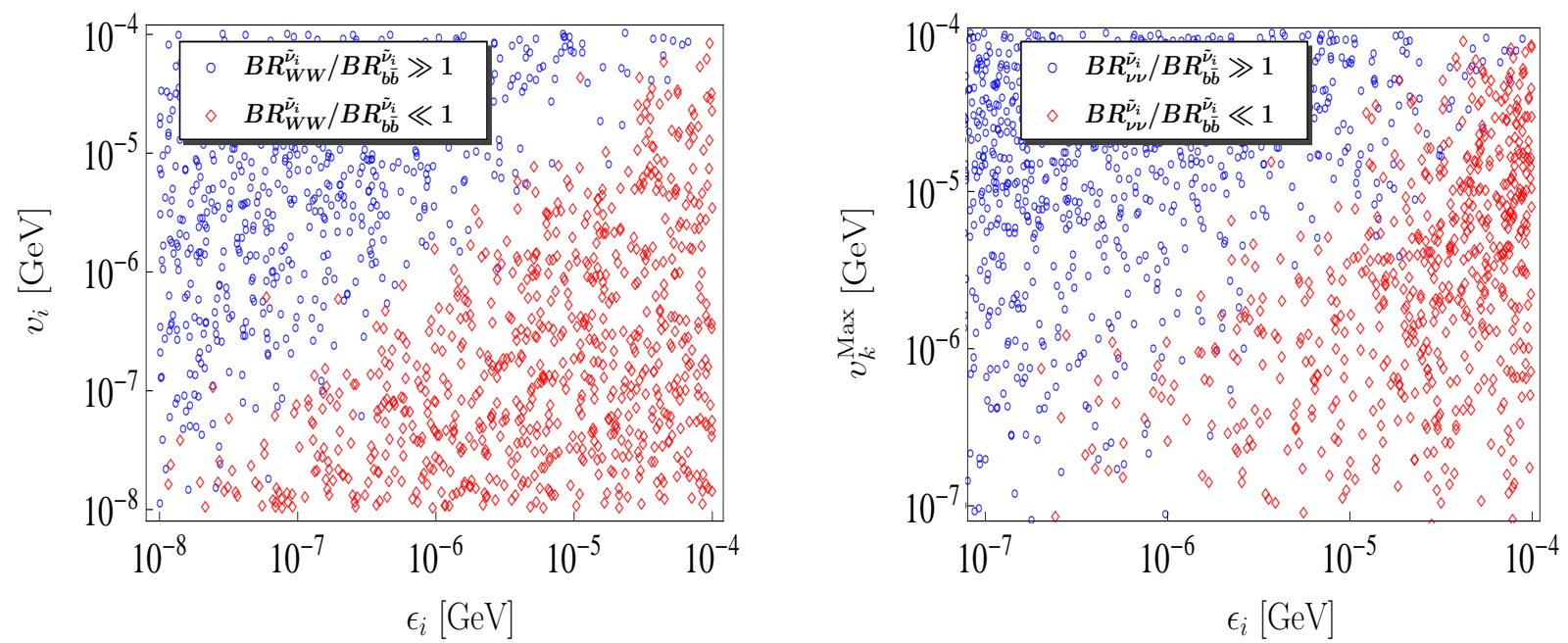

Figure 5: Results of a scan over SUSY parameters, as shown in the text, comparing the sneutrino decay width into $W^{+} W^{-}$and $\nu \nu$ with the $b \bar{b}$ final state. It has been assumed that $B R p V$ does not saturate neutrino masses. Blue points indicate where $B R_{W W}^{\tilde{\nu}_{i}} / B R_{b \bar{b}}^{\tilde{\nu}_{i}} \gg 1$ (left-hand side plot) or $B R_{\nu \nu}^{\tilde{\nu}_{i}} / B R_{b \bar{b}}^{\tilde{\nu}_{i}} \gg 1$ (right-hand side plot) while the red points the opposite. The invisible mode involves a sum over final state lepton flavors, and so its importance is determined by the relative size between $\epsilon_{i}$ and any of the three sneutrino vevs. The vertical axes in the right-hand side plot corresponds to the largest sneutrino vev obtained when randomly scanning the parameter space. These plots therefore show an important general result: the $W^{+} W^{-}$and invisible modes have larger branching fractions than the $b \bar{b}$ mode only when the sneutrino vev is much larger than its corresponding bilinear $\epsilon$ parameter.

$b \bar{b}$ mode, which was typically considered as the dominate mode [25].

We begin with a very general study ignoring all neutrino constrains (SUSY parameters can always be chosen in such a way so that BRpV does not saturate the neutrino masses) and exploring in the $\epsilon_{i}-v_{i}$ parameter space where the aforementioned modes dominate the $b \bar{b}$ decays of $\tilde{\nu}_{i}$. Without neutrino constraints, these decay properties are independent of generation. We scan over the following values:

$$
\begin{gathered}
\tan \beta=2-50, \quad m_{A}=500-1000 \mathrm{GeV}, \quad m_{\tilde{\nu}_{i}}=161-500 \mathrm{GeV}, \\
M_{1,2}=500-1000 \mathrm{GeV}, \quad v_{i}=10^{-8}-10^{-4} \mathrm{GeV}, \quad \epsilon_{i}=10^{-8}-10^{-4} \mathrm{GeV},
\end{gathered}
$$

and $\mu$ below $1000 \mathrm{GeV}$ but large enough so that the sneutrino is the LSP. We also use $m_{h}=125 \mathrm{GeV}$ as suggested by recent LHC results. For $W^{+} W^{-}$and $\nu \nu$ modes the results are displayed in figure 5 where blue dots indicate the points at which these channels have partial decay widths much larger than the $b \bar{b}$ mode, while the red points show the opposite. It is striking that the ratio of the partial widths are relatively independent of R-parity conserving SUSY parameters and depend only on the BRpV parameters. From this figure, one can conclude that the $W^{+} W^{-}$and the $\nu \nu$ partial widths dominate the $b \bar{b}$ width only when the sneutrino vev is much larger than the bilinear mixing parameter 
and, as will be shown below, this condition holds for the other heavy SM final states. Couple this with the main result of the previous section: satisfying neutrino masses solely through $\mathrm{BRpV}$ means that $v_{i} \gg \epsilon_{i}$ can only be satisfied in one generation, indicates that heavy final states as well as invisible modes can only have branching fractions larger than the $b \bar{b}$ mode for one generation of sneutrinos.

We now turn to the discussion of the different lepton flavor final states. These modes $\left(\tilde{\nu}_{i} \rightarrow l_{i}^{+} l_{k}^{-}\right)$, being induced by chargino charged lepton mixing, are mainly controlled by the bilinear mixing parameters for the flavor of the final lepton which differs from the sneutrino flavor (see eq. (34) and (60)) i.e. $\epsilon_{k} / \mu$ not $\epsilon_{i} / \mu$. The $b \bar{b}$ mode, on the other hand, is governed by $\epsilon_{i} / \mu$ (same flavor as the sneutrino) if $\epsilon_{i} \gg v_{i}$, or $v_{i} / v$, if $\epsilon_{i} \ll v_{i}$.

Therefore, when $\epsilon_{i} \gg v_{i}$ (region where $b b$ modes dominate $W W$ ), neutrino constraints imply all three $\epsilon$ s are of the same order (see Fig. 3). Therefore the different lepton flavor final states become subdominant to the $b b$ modes due to charged lepton Yukawa suppression. In contrast, when $\epsilon_{i} \ll v_{i}$ (WW modes dominate $b b$ modes) the different lepton flavor final states can have larger partial widths than the $b \bar{b}$ mode provided $\left[\left(\epsilon_{k} / \mu\right) /\left(v_{i} / v\right)\right]^{2}$ exceeds the corresponding Yukawa suppression $\left(h_{i}^{E} / h_{b}\right)^{2}$, which turns out to be possible only for second and third generation sneutrinos as shown in Fig. 3, where it can be seen that $\left[\left(\epsilon_{k} / \mu\right) /\left(v_{i} / v\right)\right]^{2}$ can be at most $10^{4}$ (note that $\left(h_{\tau} / h_{b}\right)^{2} \sim 10^{-1},\left(h_{\mu} / h_{b}\right)^{2} \sim 10^{-3}$ while $\left.\left(h_{e} / h_{b}\right)^{2} \sim 10^{-8}\right)$. As in the case of heavy SM and invisible modes, these different lepton flavor final states can dominant over the $b \bar{b}$ final state only in a single sneutrino generation.

We continue by considering the specific SUSY point discussed in the last section and examining first and third generation sneutrino decays into $b \bar{b}, W^{+} W^{-}, \sum_{j, k} \nu_{j} \nu_{k}$ and different lepton flavor modes assuming that $\mathrm{BRpV}$ is solely responsible for neutrino masses. We have also calculated the partial decay widths for the $h^{0} h^{0}$ and $Z^{0} Z^{0}$ final states, finding that they have a similar dependence on $\epsilon_{3} / \mu$ and are of the same order as the $W^{+} W^{-}$ mode (the $t \bar{t}$ channel is suppressed due to the off-shell top for this sneutrino mass). The results are displayed in figure 6 versus $\epsilon_{3} / \mu$, where it can be seen that $\tilde{\nu}_{1}$ exhibits the usual bahavior quoted in the literature, that is to say, a dominant $b \bar{b}$ mode followed by a slightly Yukawa suppressed $\tau \bar{\tau}$ mode and strongly suppressed $\nu \nu, W W, \bar{e} \mu$ and $\bar{e} \tau$ final states. The huge supression of the different lepton flavor mode, $\bar{e} \tau$, in the small $\epsilon_{3}$ region is due to the combined effect of the electron Yukawa suppression and the smallness of $v_{3} / \epsilon_{1}$. The $\bar{e} \mu$ final state being controlled by $\epsilon_{2} / \mu$ involves only the electron Yukawa suppression, and so it has a larger partial decay width than the $\bar{e} \tau$ mode.

The situation for $\tilde{\nu}_{3}$, however, is quite different. In the region of large $\epsilon_{3}$ it features a $b \bar{b}$ dominant mode and apart from the $\bar{\tau} e$ mode it behaves like $\tilde{\nu}_{1}$. In the region of small $\epsilon_{3}$, in contrast, the "non-conventional" modes $\bar{\tau} \mu(\bar{\tau} e), \nu \nu, W^{+} W^{-}, h^{0} h^{0}$ and $Z Z$ have the largest partial widths by far, greatly surpassing the $b \bar{b}$ final state. In particular, the different lepton flavor channels exceed this mode by more than two-orders of magnitude, thus drastically changing the sneutrino LSP phenomenology.

Some words are in order as regards the size of the $\tau \bar{\tau}$ partial width in $\tilde{\nu}_{3}$ decays. This decay mode is a unequivocal signal of BRpV models, but its size is strongly dependent on the nature of the bilinear parameters. In R-parity violating models satisfying the 

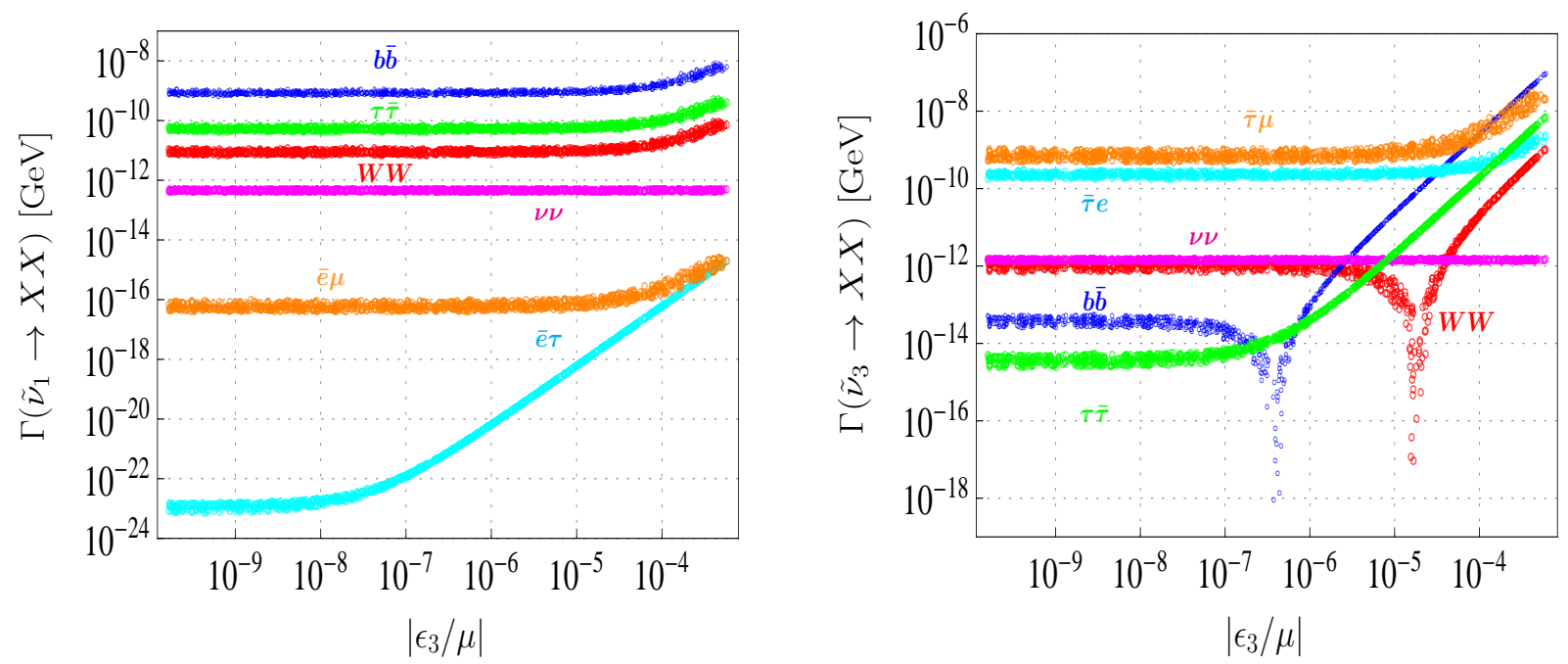

Figure 6: Partial decay widths for $\tilde{\nu}_{1}$ (left) and $\tilde{\nu}_{3}$ (right) to $b \bar{b}, W W, \tau \bar{\tau}, \sum_{j, k} \nu_{j} \nu_{k}$, eq, $e \mu$ and $\tau \mu$ final states as a function of $\left|\epsilon_{3} / \mu\right|$. BRp V parameters have been fixed by using neutrino oscillation data (see the text for more details). The remaining heavy $S M$ modes, hh and $Z Z$, have the same behavior and are of the same order than the $W W$ mode (are not shown to avoid crowding the plots). Decays of $\tilde{\nu}_{2}$ are not shown since they are very similar to those of $\tilde{\nu}_{1}$.

conditions $m_{H_{d}}^{2}=m_{L_{i}}^{2}$ and $B=B_{i}$ at certain high energy scale, the bilinears can be rotated away. They are, however, subsequently induced at the TeV scale by RGE running effects $[50,51,52]$ therefore inducing the $\tau \bar{\tau}$ mode, but with a quite suppressed partial width (typically several orders of magnitude smaller than that of the $b \bar{b}$ channel). In a MSSM based analysis, as the one performed here, where all the BRpV parameters are treated as free parameters subject only to phenomenological constraints this is not necessarily the case, as demonstrated in the right-hand side plot in Fig. 6.

Figure 6 reinforces the arguments given in the previous paragraphs. Reiterating: even if bilinear R-parity violation accounts for neutrino data, the $b \bar{b}$ channel is not necessarily the dominant decay mode. We have checked that for third and second generation sneutrino LSPs the different lepton flavor final states dominate $\left(\bar{\tau} e, \bar{\tau} \mu\right.$ for $\tilde{\nu}_{3}$ and $\bar{\mu} e, \bar{\mu} \tau$ for $\left.\tilde{\nu}_{2}\right)$ while first generation sneutrinos decays are driven by invisible and heavy SM modes ( $h h$ and $W W)$. As already pointed out and exemplified for $\tilde{\nu}_{3}$ in Fig. 6, these decays can dominate only in a single sneutrino generation. So, observing at least two different sneutrino flavors decaying to the aforementioned modes $^{12}$ (something that turns out to be viable if the sneutrino mass splittings do not allow the heavier sneutrinos to decay into the LSP) will prove that BRpV does not account for neutrino data, because this would require $v_{i} \gg \epsilon_{i}$ for those generations, which is not possible if neutrino physics is determined solely by BRpV, see Fig. 4.

\footnotetext{
${ }^{12}$ In principle for sneutrinos originating from chargino decays the corresponding sneutrino flavor could be tagged by the associated lepton flavor [25].
} 

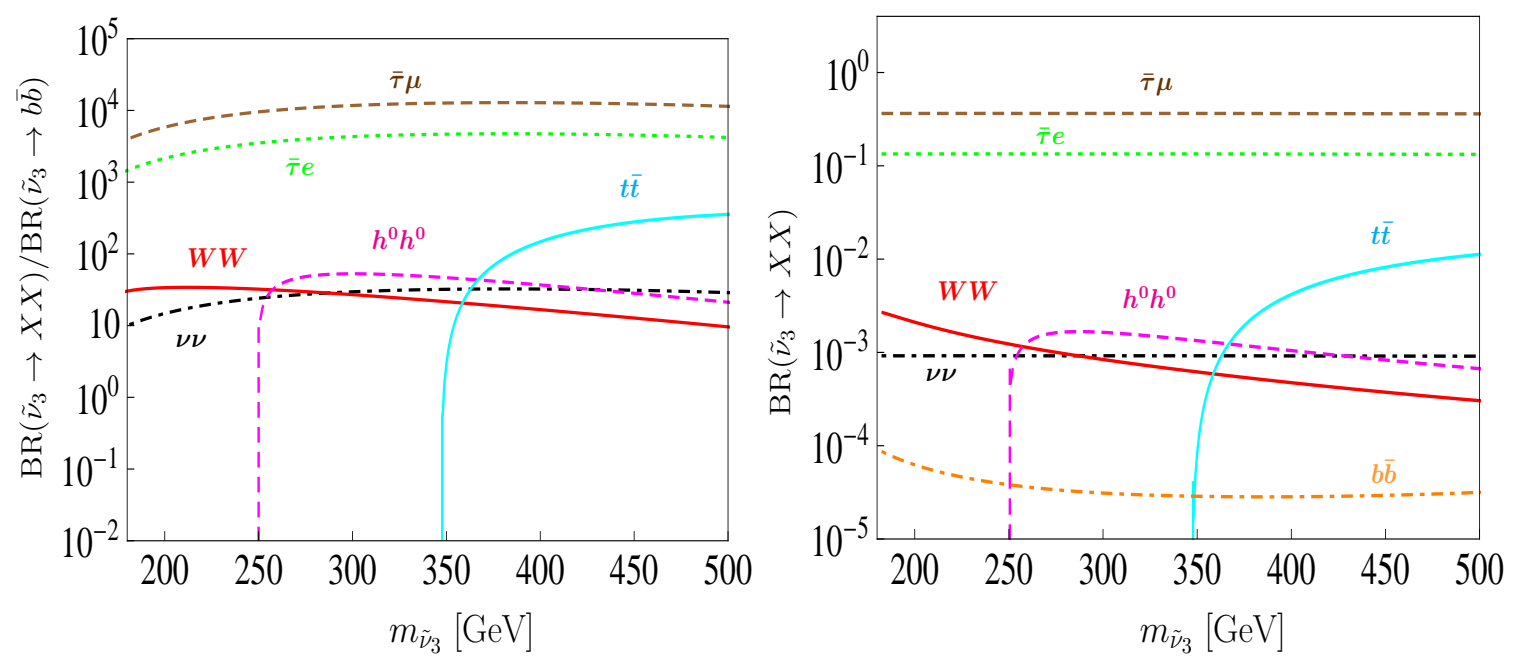

Figure 7: Left plot: decay branching fractions for different lepton flavor ( $\bar{\tau} e$ and $\bar{\tau} \mu)$, invisible $\left(\sum_{j, k} \nu_{j} \nu_{k}\right), W W$, hh and $t \bar{t}$ final states normalized to $B R\left(\tilde{\nu}_{3} \rightarrow b \bar{b}\right)$ as a function of $m_{\tilde{\nu}_{3}}$. Right plot: decay branching ratios for dominant (excluding $Z Z$ ) and $b \bar{b}$ modes as a function of $m_{\tilde{\nu}_{3}}$. The branching ratios have been calculated in the small $\epsilon_{3}$ region $\left(\left|\epsilon_{3} / \mu\right|=10^{-9}\right)$ and for bilinear $R$-parity breaking parameters fixed by neutrino parameters according to their best fit point values [44, 45, 46]. Both plots show the main result of this paper: sneutrino LSP decays are not necessarily dominated by the $b \bar{b}$ mode.

Focusing further on those final states of $\tilde{\nu}_{3}$ decays (excluding the $Z Z$ mode since it behaves like the $W W$ mode and is smaller by an order of $1 / 2$ ), we study $\tilde{\nu}_{3}$ decays as a function of $m_{\tilde{\nu}_{3}}$ in Figure 7 allowing a $\tilde{\nu}_{3}$ heavy enough so that the $t \bar{t}$ channel is also open. All the R-parity conserving parameters have been fixed according to the supersymmetric point used in the previous section. The R-parity breaking parameters have been fixed by adjusting neutrino observables to their best fit point values $[44,45,46]$ and $\epsilon_{3}=\mu \times 10^{-9}$. We have checked that the values are insensitive to changes in $\tan \beta$ in the range $[2,30]$. Off-shell calculations of the Higgs and top states have been neglected since the different lepton flavor final states dominant over the full sneutrino mass range. Both plots support our claim: even if BRpV is the sole generator of neutrino masses a sneutrino LSP does not necessarily decay predominantly into $b \bar{b}$ final states.

One can extrapolate from Figure 6 the behavior of the R-parity violating sneutrino decays for the first two generations, in the analogous region $\left\langle\tilde{\nu}_{1,2}\right\rangle \gg \epsilon_{1,2}$. In these cases, only the leptonic different flavor states shift and they do so by a factor of approximately $\left(\boldsymbol{h}_{\mu}^{\boldsymbol{E}} / \boldsymbol{h}_{\tau}^{\boldsymbol{E}}\right)^{2}$ for $\tilde{\nu}_{2} \rightarrow \mu \bar{\tau}$ and $\left(\boldsymbol{h}_{e}^{\boldsymbol{E}} / \boldsymbol{h}_{\tau}^{\boldsymbol{E}}\right)^{2}$ for $\tilde{\nu}_{1} \rightarrow e \mu$. Therefore, $\tilde{\nu}_{2}$ decays will still be dominated by different flavor leptonic final states, while $\tilde{\nu}_{1}$ will decay predominately into heavy SM states and neutrinos.

Relaxing the assumption that bilinear R-parity violation is the sole generator of neutrino masses (as in the second case discussed in section 4.3) will not drastically change the results in this section. This is because the relative importance of the ratios $\mathrm{BR}_{X X}^{\tilde{\nu}_{i}} / \mathrm{BR}_{b \bar{b}}^{\tilde{\nu}_{i}}$ is determined by $v_{i} \gg \epsilon_{i}$. However, a quantity that becomes relevant in this case is the 


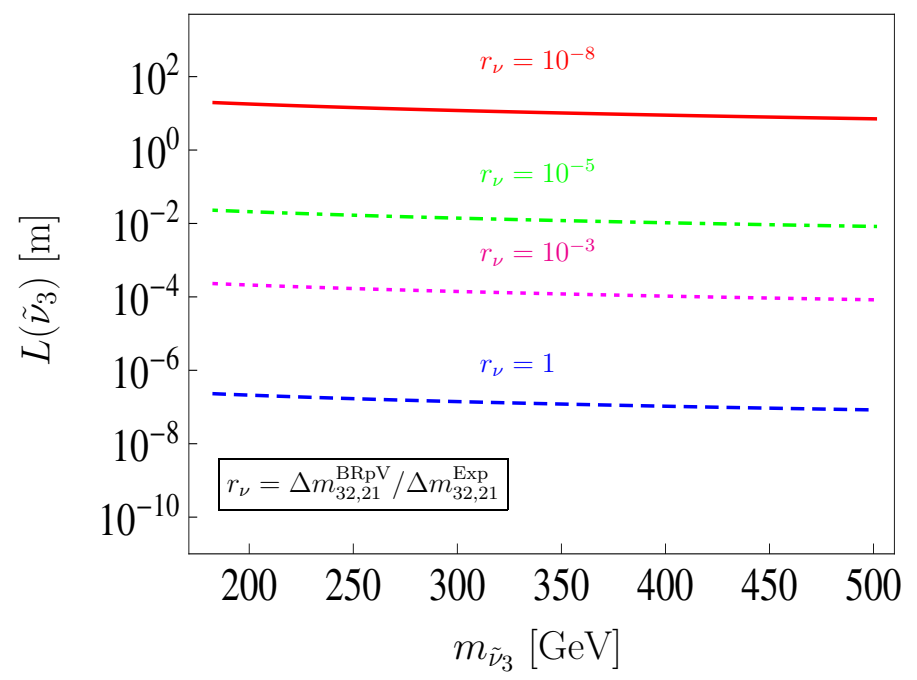

Figure 8: Sneutrino decay length for different choices of the bilinear $R$-parity breaking parameters. The BRpV contribution to the atmospheric and solar mass scales is specified

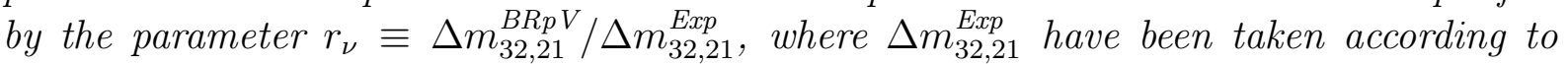
their best fit point values [44, 45, 46].

sneutrino decay length $L\left(\tilde{\nu}_{i}\right)$, since the size of the bilinear R-parity breaking parameters can be small enough to cause the sneutrino to decay outside of the detector. Neglecting the Lorentz boost factor, we have found that as long as the R-parity violating parameters fit the neutrino data, in general $L\left(\tilde{\nu}_{i}\right)$ is well below $1 \mathrm{~mm}$ (inline with [27]). We have also checked that if $r_{\nu}=\Delta m_{32,21}^{\mathrm{BRpV}} / \Delta m_{32,21}^{\mathrm{Exp}}=10^{-5}$ the decay length is generically below $\sim 10 \mathrm{~cm}$. Note that values of $r_{\nu}$ in the range $\left[10^{-3}, 1\right]$ might be in conflict with neutrino data when the contributions from the mechanism responsible for neutrino masses are taken into account (for example a standard seesaw). Once the contributions to the atmospheric and solar mass scales fall below $r_{\nu}=10^{-5}$ the decay length can eventually exceed $1 \mathrm{~m}$ almost independently of the sneutrino mass (as expected due to the large partial decay widths the different lepton flavor modes have). Figure 8 shows the decay length for $r_{\nu}=1,10^{-3}, 10^{-5}, 10^{-8}$.

Thus far, this section has focused on sneutrino decays. At this point, we briefly comment on production. If the sneutrino is indeed the LSP, all produced supersymmetric particles produced will cascade decay down to it. The dominate production depends on the relative masses, with strong production [53] dominating unless the gluinos and squarks are too heavy, in which case relatively light sneutrinos can still be pair produced through electroweak processes. Regardless, the parameter region explored in this paper yields compelling signals since the dominate different flavor leptonic final states would have little background if the intermediate sneutrino can be reconstructed. Meanwhile, the $b \bar{b}$ mode corresponding to the parameter space typically considered in the literature would suffer from larger QCD background and would therefore be more difficult to detect. Furthermore, if the BRpV contributions to neutrino masses are subdominant to some 
other mechanism, sneutrino decays could involve large displaced vertices (see Fig. 8) thus rendering its identification even more plausible.

\section{Conclusions}

$\mathrm{BRpV}$ is a good effective theory for models of spontaneous R-parity violation, in which proton decay as well as the number of new parameters is under control compared to explicit R-parity violation. In the presence of BRpV, sneutrino LSP decays can in some cases resemble a heavy Higgs. However, as we have discussed, sneutrinos (third and second generation) can dominantly decay to different-flavor charged leptons final states or heavy SM modes (first generation), thus completely altering the "conventional" sneutrino LSP phenomenology driven by the $b \bar{b}$ mode.

We have studied these decays into $W^{+} W^{-}, Z Z, h^{0} h^{0}, t \bar{t}, \nu \nu$ and $l_{i}^{+} l_{k}^{-}(i \neq k)$ and found that long as $\epsilon_{i} \ll v_{i}$, they have larger branching ratios than the $b \bar{b}$ mode. As discussed in section 4.3, for models where the BRpV parameters are fixed by neutrino data, this is possible only for a single sneutrino flavor, due to the constraints from the solar sector. Therefore, if 2 or more sneutrino generations are degenerate enough to decay via $\mathrm{BRpV}$ into these states one could rule out $\mathrm{BRpV}$ as the sole source of neutrino masses.

For models where the bilinear R-parity breaking parameters do not contribute significantly to neutrino masses, the constraint $\epsilon_{i} \ll v_{i}$ for all flavors is viable. Accordingly, in these BRpV models large sneutrino LSP branching fractions into either $l_{i}^{+} l_{k}^{-}$or $W^{+} W^{-}$, $Z Z, h^{0} h^{0}$ and $t \bar{t}$ are possible. In this case, however, special attention has to be paid to the sneutrino decay length $L\left(\tilde{\nu}_{i}\right)$. We calculated $L\left(\tilde{\nu}_{i}\right)$, finding that as long as the BRpV contributions to neutrino masses are not below $\sim 10^{-3 \%}$ the decay length is generically below $\sim 10 \mathrm{~cm}$.

Regardless of these considerations, sneutrino decays into different-flavor charged leptons or heavy SM final states $\left(l_{i}^{+} l_{k}^{-}\right.$or $W^{+} W^{-}, Z Z, h^{0} h^{0}$ and $\left.t \bar{t}\right)$ are an interesting phenomenological possibility that has not received much attention before. Because BRpV only affects the decays of the LSP, assuming a sneutrino LSP means that SUSY events could cascade decay to either different flavor charged leptons or two pairs of heavy SM states, which constitutes a unique and novel SUSY signature.

\section{Acknowledgments}

We want to thank Martin Hirsch for his comments in the early stage of this project and Werner Porod for SPheno support. SS would also like to thank Pavel Fileviez Perez for comments. DAS is supported by a Belgian FNRS postdoctoral fellowship. DR has been partially supported by Sostenibilidad-UdeA and COLCIENCIAS through the grant number 111556934918. 


\section{References}

[1] G. Aad et al. [ATLAS Collaboration], Phys. Lett. B 716 (2012) 1 [arXiv:1207.7214 [hep-ex]].

[2] S. Chatrchyan et al. [CMS Collaboration], Phys. Lett. B 716 (2012) 30 [arXiv:1207.7235 [hep-ex]].

[3] R. Barbier, C. Berat, M. Besancon, M. Chemtob, A. Deandrea, E. Dudas, P. Fayet and S. Lavignac et al., Phys. Rept. 420, 1 (2005) [hep-ph/0406039].

[4] S. Chatrchyan et al. [CMS Collaboration], arXiv:1206.3949 [hep-ex].

[5] G. Aad et al. [ATLAS Collaboration], Phys. Lett. B 709, 137 (2012) [arXiv:1110.6189 [hep-ex]].

[6] L. M. Carpenter, D. E. Kaplan and E. -J. Rhee, Phys. Rev. Lett. 99, 211801 (2007) [hep-ph/0607204].

[7] L. M. Carpenter, D. E. Kaplan and E. J. Rhee, arXiv:0804.1581 [hep-ph].

[8] P. W. Graham, D. E. Kaplan, S. Rajendran and P. Saraswat, JHEP 1207, 149 (2012) [arXiv:1204.6038 [hep-ph]].

[9] C. S. Aulakh and R. N. Mohapatra, Phys. Lett. B 119 (1982) 136.

[10] M. J. Hayashi and A. Murayama, Phys. Lett. B 153 (1985) 251.

[11] R. N. Mohapatra, Phys. Rev. Lett. 56 (1986) 561.

[12] J. M. Mira, E. Nardi, D. A. Restrepo and J. W. F. Valle, Phys. Lett. B 492, 81 (2000) [hep-ph/0007266].

[13] D. Aristizabal Sierra, D. Restrepo and O. Zapata, Phys. Rev. D 80, 055010 (2009) [arXiv:0907.0682 [hep-ph]].

[14] C. S. Aulakh, A. Melfo, A. Rasin and G. Senjanovic, Phys. Lett. B 459 (1999) 557 [hep-ph/9902409].

[15] C. S. Aulakh, B. Bajc, A. Melfo, A. Rasin and G. Senjanovic, Nucl. Phys. B 597 (2001) 89 [hep-ph/0004031].

[16] P. Fileviez Perez and S. Spinner, Phys. Lett. B 673 (2009) 251 [arXiv:0811.3424 [hep-ph]].

[17] V. Barger, P. Fileviez Perez and S. Spinner, Phys. Rev. Lett. 102 (2009) 181802 [arXiv:0812.3661 [hep-ph]]. 
[18] M. Ambroso and B. Ovrut, JHEP 0910 (2009) 011 [arXiv:0904.4509 [hep-th]]; M. Ambroso and B. A. Ovrut, Int. J. Mod. Phys. A 25 (2010) 2631 [arXiv:0910.1129 [hep-th]].

[19] P. Fileviez Perez and S. Spinner, Phys. Rev. D 83 (2011) 035004 [arXiv:1005.4930 [hep-ph]].

[20] A. Masiero and J. W. F. Valle, Phys. Lett. B 251, 273 (1990);

[21] W. Buchmuller, L. Covi, K. Hamaguchi, A. Ibarra and T. Yanagida, JHEP 0703, 037 (2007) [hep-ph/0702184 [HEP-PH]].

[22] F. Takayama and M. Yamaguchi, "Gravitino dark matter without R-parity," Phys. Lett. B 485, 388 (2000) [hep-ph/0005214].

[23] S. Dimopoulos, D. Eichler, R. Esmailzadeh and G. D. Starkman, Phys. Rev. D 41, 2388 (1990).

[24] A. Bartl, M. Hirsch, T. Kernreiter, W. Porod and J. W. F. Valle, JHEP 0311, 005 (2003) [hep-ph/0306071].

[25] D. Aristizabal Sierra, M. Hirsch and W. Porod, JHEP 0509, 033 (2005) [hep$\mathrm{ph} / 0409241]$.

[26] W. Porod, M. Hirsch, J. Romao and J. W. F. Valle, Phys. Rev. D 63, 115004 (2001) [hep-ph/0011248].

[27] M. Hirsch and W. Porod, Phys. Rev. D 68, 115007 (2003) [hep-ph/0307364].

[28] S. Roy, B. Mukhopadhyaya and, Phys. Rev. D 55, 7020 (1997) [hep-ph/9612447].

[29] B. Mukhopadhyaya, S. Roy, F. Vissani and , Phys. Lett. B 443, 191 (1998) [hep$\mathrm{ph} / 9808265]$.

[30] S. Bar-Shalom, G. Eilam and B. Mele, Phys. Rev. D 64, 095008 (2001) [hep$\mathrm{ph} / 0106053]$.

[31] Y. Grossman, H. E. Haber and , Phys. Rev. D 59, 093008 (1999) [hep-ph/9810536].

[32] M. Hirsch, M. A. Diaz, W. Porod, J. C. Romao and J. W. F. Valle, Phys. Rev. D 62, 113008 (2000) [Erratum-ibid. D 65, 119901 (2002)] [hep-ph/0004115].

[33] M. A. Diaz, M. Hirsch, W. Porod, J. C. Romao and J. W. F. Valle, Phys. Rev. D 68, 013009 (2003) [Erratum-ibid. D 71, 059904 (2005)] [hep-ph/0302021].

[34] M. Nowakowski and A. Pilaftsis, Nucl. Phys. B 461, 19 (1996) [hep-ph/9508271].

[35] M. Hirsch and J. W. F. Valle, Nucl. Phys. B 557, 60 (1999) [hep-ph/9812463]. 
[36] M. Drees and M. M. Nojiri, Phys. Rev. D 45, 2482 (1992).

[37] F. de Campos, M. A. Garcia-Jareno, A. S. Joshipura, J. Rosiek, J. W. F. Valle, Nucl. Phys. B 451, 3 (1995) [hep-ph/9502237].

[38] A. Djouadi, J. Kalinowski and P. M. Zerwas, Z. Phys. C 70, 435 (1996) [hepph/9511342].

[39] A. Djouadi, Phys. Rept. 457, 1 (2008) [hep-ph/0503172].

[40] E. Ma, Mod. Phys. Lett. A 17, 1259 (2002) [hep-ph/0205025].

[41] D. Aristizabal Sierra, M. Hirsch, J. W. F. Valle and A. Villanova del Moral, Phys. Rev. D 68, 033006 (2003) [hep-ph/0304141].

[42] D. K. Ghosh, G. Senjanovic and Y. Zhang, Phys. Lett. B 698, 420 (2011) [arXiv:1010.3968 [hep-ph]].

[43] V. Barger, P. Fileviez Perez and S. Spinner, Phys. Lett. B 696, 509 (2011) [arXiv:1010.4023 [hep-ph]].

[44] D. V. Forero, M. Tortola and J. W. F. Valle, Phys. Rev. D 86, 073012 (2012) [arXiv:1205.4018 [hep-ph]].

[45] M. C. Gonzalez-Garcia, M. Maltoni, J. Salvado and T. Schwetz, arXiv:1209.3023 [hep-ph].

[46] G. L. Fogli, E. Lisi, A. Marrone, D. Montanino, A. Palazzo and A. M. Rotunno, Phys. Rev. D 86, 013012 (2012) [arXiv:1205.5254 [hep-ph]].

[47] G. Passarino and M. J. G. Veltman, Nucl. Phys. B 160, 151 (1979).

[48] W. Porod, Comput. Phys. Commun. 153, 275 (2003) [hep-ph/0301101]; W. Porod and F. Staub, Comput. Phys. Commun. 183, 2458 (2012) [arXiv:1104.1573 [hep-ph]].

[49] J. Abdallah et al. [DELPHI Collaboration], Eur. Phys. J. C 31, 421 (2003) [hepex/0311019].

[50] B. de Carlos and P. L. White, Phys. Rev. D 55, 4222 (1997) [hep-ph/9609443].

[51] B. de Carlos and P. L. White, Phys. Rev. D 54, 3427 (1996) [hep-ph/9602381].

[52] E. Nardi, Phys. Rev. D 55, 5772 (1997) [hep-ph/9610540].

[53] M. Kramer, A. Kulesza, R. van der Leeuw, M. Mangano, S. Padhi, T. Plehn, X. Portell and, arXiv:1206.2892 [hep-ph]. 